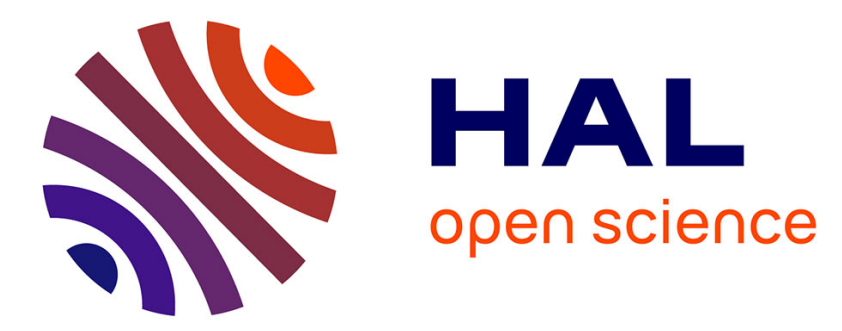

\title{
Redox switching of Prussian blue thin films investigated by ac-electrogravimetry
}

Loan To Thi Kim, Claude Gabrielli, Hubert Perrot, J. Garcia-Jareno, F.

Vicente

\section{- To cite this version:}

Loan To Thi Kim, Claude Gabrielli, Hubert Perrot, J. Garcia-Jareno, F. Vicente. Redox switching of Prussian blue thin films investigated by ac-electrogravimetry. Electrochimica Acta, 2012, 84, pp.35-48. 10.1016/j.electacta.2012.06.049 . hal-00790548

\section{HAL Id: hal-00790548 \\ https: / hal.sorbonne-universite.fr/hal-00790548}

Submitted on 22 Apr 2015

HAL is a multi-disciplinary open access archive for the deposit and dissemination of scientific research documents, whether they are published or not. The documents may come from teaching and research institutions in France or abroad, or from public or private research centers.
L'archive ouverte pluridisciplinaire HAL, est destinée au dépôt et à la diffusion de documents scientifiques de niveau recherche, publiés ou non, émanant des établissements d'enseignement et de recherche français ou étrangers, des laboratoires publics ou privés. 


\title{
Redox switching of Prussian Blue Thin Films Investigated by ac-electrogravimetry.
}

\author{
L. To Thi Kim ${ }^{1,2}$, C. Gabrielli ${ }^{1,2}$, H. Perrot ${ }^{1,2, *}$, \\ J. Garcia-Jareno ${ }^{3}$, F Vicente ${ }^{3}$. \\ 1-CNRS, UPR 15, LISE, F-75252, Paris, France \\ 2-UPMC Univ. Paris 06, UPR15, LISE, F- 75252 Paris, France \\ 3-Depto de Quimica-Fisica, Univ. Valencia, Burjassot, Spain
}

\begin{abstract}
In this paper, the redox switching behaviour of Prussian Blue (PB) in $\mathrm{KCl}$ environment at $\mathrm{pH} 2.5$ and 5.4 was investigated by using $a c$-electrogravimetry in a potential range around the conversion of PB to ES (Everitt Salt). This technique allows the electrochemical impedance and a mass-potential transfer function to be simultaneously measured. From the impedance the charge-potential transfer function is calculated. A model of the PB film, is presented, it takes into account the porous structure of the material, the insertion of two cations on the pore wall, and the electronic charge transfer from the electrode. A fitting procedure was employed to obtain the kinetic parameters.

Attractive information extracted from the impedance concerns the porosity of the PB film which is related to the zeolitic nature of the film. The resistivity of the pore depends obviously on the electrolyte conductance and changes with respect to the potential with a minimum, in the vicinity of the $\mathrm{PB} \leftrightarrow \mathrm{ES}$ conversion potential-

$A c$-electrogravimetry and the charge-potential transfer function gave essentially information on the kinetics of the ionic transfer. Thanks to the model of electroactive porous films and the digital fitting procedure, for the first time, the two cations $\mathrm{K}^{+}$and $\mathrm{H}_{3} \mathrm{O}^{+}$are identified with well separated kinetics for each cation. Several attractive informations were reached. First, it confirms that ionic transfer at the film/electrolyte interface limits the kinetics of the film reduction or oxidation as electronic transfer at the electrode/film interface is faster. Moreover, the rates of the electronic and ionic transfer kinetics vary with the potential. They are faster around the potential where the $\mathrm{PB} \leftrightarrow \mathrm{ES}$ conversion occurs. It was confirmed that charge compensation occurs only by $\mathrm{K}^{+}$and $\mathrm{H}_{3} \mathrm{O}^{+}$movements whatever the $\mathrm{pH}$ of the solution. However, $\mathrm{H}_{3} \mathrm{O}^{+}$transfer kinetics is faster than $\mathrm{K}^{+}$transfer, whatever the $\mathrm{KCl}$ concentration, and the role of $\mathrm{H}_{3} \mathrm{O}^{+}$is more important for large $\mathrm{KCl}$ concentrations where the
\end{abstract}


process is more limited by the kinetics of the hydronium ions. In addition, in these situations, even if ionic transfer is also accelerated the global charge transfer is nevertheless more controlled by electronic transfer

KEYWORDS: $a c$-electrogravimetry; Prussian Blue; Porosity; ionic transfer; electronic transfer

* Corresponding author 


\section{1 - INTRODUCTION}

Prussian Blue (PB) or iron hexacyanoferrate is considered as the first synthesised coordination compound. Although it has been studied for 270 years, it is still a research topic of high interest because of its numerous interesting properties as electrochromism, ion exchanger, and photomagnetic properties which allow applications in electrocatalysis, electrochromic or electronic devices, and batteries. PB films can be generated by chemical [12] or electrochemical [3-4] ways, often from an aqueous solution of a $\mathrm{FeCl}_{3}$ and $\mathrm{K}_{3} \mathrm{Fe}(\mathrm{CN})_{6}$ mixture. PB was deposited at an electrode surface for the first time by V.D. Neff [5] The films obtained after the deposit are under the so-called insoluble form with formula $\mathrm{Fe}_{4}{ }^{3+}\left[\mathrm{Fe}^{2+}(\mathrm{CN})_{6}\right]_{3} \cdot \mathrm{mH}_{2} \mathrm{O}$. The films are converted under the so-called soluble form $\mathrm{KFe}^{3+}\left[\mathrm{Fe}^{2+}(\mathrm{CN})_{6}\right] \cdot \mathrm{mH}_{2} \mathrm{O}$ after a few voltammetric cycles in a $\mathrm{KCl}$ solution.

The first structure of PB has been proposed by Keggin and Miles [6] from X rays studies. In the $\mathrm{Fe}_{4}{ }^{3+}\left[\mathrm{Fe}^{2+}(\mathrm{CN})_{6}\right]_{3} \cdot \mathrm{mH}_{2} \mathrm{O}$ crystalline structure, $\mathrm{Fe}^{3+}$ ions (high spin iron) and $\mathrm{Fe}^{2+}$ iron (low spin iron) are alternatively localized on the centered faces of the cubic lattice in a way that the $\mathrm{Fe}^{3+}$ ions are surrounded by nitrogen atoms and the $\mathrm{Fe}^{2+}$ ions are surrounded by carbon atoms. During the conversion between the insoluble and soluble forms, the film loses a quarter of the $\mathrm{Fe}^{3+}$ ions (high spin iron) which are substituted by the $\mathrm{K}^{+}$cations. In addition, it has been shown that the soluble structure contains between 14 and 16 water molecules by elementary mesh unit [7]. In the PB film, two types of water molecules have been observed by neutron diffractometry [8]: 6 water molecules are coordinated with the $\mathrm{Fe}(\mathrm{CN})_{6}{ }^{4+}$ vacancies and the others occupy interstitial sites without any coordination. Ganguli et al. [9] have studied the dehydration process of PB by thermogravimetry. They have identified three different hydration states and an anhydrous one. The decomposition occurs at temperature greater than $150^{\circ} \mathrm{C}$. The hydration degree of the films depends on the temperature and humidity of the environment. It was observed that about $90 \%$ of water is eliminated by a $150^{\circ} \mathrm{C}$ heating. When dehydrated $\mathrm{PB}$ is reexposed to ambient air, it takes again the same hydration amount.

The PB soluble film [10] is reduced to the uncoloured form, called Everitt salt (ES), in a $\mathrm{KCl}$ solution:

$$
\underset{\text { PB }}{\mathrm{KFe}^{3+} \mathrm{Fe}^{2+}(\mathrm{CN})_{6}+e^{-}+\mathrm{K}^{+} \leftrightarrow \underset{\mathrm{ES}}{\mathrm{K}_{2}} \mathrm{Fe}^{2+} \mathrm{Fe}^{2+}(\mathrm{CN})_{6}}
$$

Itaya et al. [10] have shown by spectroelectrochemistry that only some cations can migrate through the PB film because of its zeolitic and nanoporous structure (pore diameter: 
$3,2 \AA$ ). Thus, this structure is permeable to cations with radii (r) less than $1,6 \AA$, such as $\mathrm{K}^{+}$(r $=1,25 \AA), \mathrm{Rb}^{+}(\mathrm{r}=1,18 \AA), \mathrm{Cs}^{+}(\mathrm{r}=1,19 \AA)$, and $\mathrm{NH}_{4}{ }^{+}(\mathrm{r}=1,25 \AA)$ but cations with greater radii such as $\mathrm{Li}^{+}(\mathrm{r}=2,39 \AA)$ and $\mathrm{Mg}^{+}(\mathrm{r}=3,47 \AA)$ are blocked. However, the $\mathrm{Na}^{+}$cation $(\mathrm{r}=$ $1.84 \AA$ ) can be also inserted in an amorphous PB film formed at larger cathodic currents. Garcia-Jareno et al. [11] have also shown by ac-electrogravimetry that the $\mathrm{Na}^{+}$cation is partially dehydrated before to be transported through the zeolitic channels of PB during an electrochemical process.

The use of an electrochemical quartz microbalance (EQCM) was very efficient to understand the ionic transfer which is involved in the redox process of a PB film. It has been shown that the insertion/expulsion process of the alcaline cation is more complicated than what is presented in Equation (1). Feldman et al. [12] have proposed that the insertion of the alcaline cation is accompanied by the proton insertion or by the expulsion of solvent, the measured apparent molar mass is the average of the proton and the alcaline cation masses. Later, by a mirage effect technique [13], the cation flux has been also found in competition with the proton flux. By using the electrochemical impedance technique, Garcia-Jareno et al. $[14,15]$ have demonstrated that protons play an important role in the kinetics of the electron transport by hopping and that the solvent contribution is necessary to accompany counter ions. Kim et al. [16] have reached the same conclusion in 2001 by cyclic electrogravimetry. These authors have reported that the insertion/expulsion process of the alcaline cation is accompanied by the transfer of free solvent $\left(\mathrm{H}_{2} \mathrm{O}\right)$ in the $\mathrm{K}_{2} \mathrm{SO}_{4} 0.5 \mathrm{M}-\mathrm{pH} 6.3$ medium. Whereas, in the same solution at $\mathrm{pH} 2.7$, the $\mathrm{K}^{+}$ion transfer is not only accompanied by solvent transfer but is also in competition with the transfer of the solvated proton. The first investigations of the $\mathrm{PB}$ films in $\mathrm{KCl}$ at $\mathrm{pH} 2.7$ by ac-electrogravimetry, [17] have allowed information on the insertion kinetics of the $\mathrm{K}^{+}$and $\mathrm{H}_{3} \mathrm{O}^{+}$cations to be obtained at various potentials. When the film is polarized in cathodic regime, only the $\mathrm{K}^{+}$cation participation was shown through the determination of a molar mass equal to $39 \mathrm{~g} \cdot \mathrm{mol}^{-1}$ but the solvated proton is mainly involved in the charge compensation towards the more positive potentials where a molar mass equal to 19 g.mole ${ }^{-1}$ was obtained. At intermediate potentials, the average molar mass changes between 19 and 39 g.mole ${ }^{-1}$. It is noticeable that in these works, the kinetics of the two cations were not separated and only a global ionic transfer was taken into account. $A c$ electrogravimetry was also recently used to examine the electrochemical behavior of hybrid inorganic-organic electroactive films [18] or bilayers films employed for PEM characterization [19]. 
This work was aimed at studying the kinetics of $\mathrm{PB}$ in $\mathrm{KCl}$ - medium at $\mathrm{pH} 2.5$ and $\mathrm{pH}$ 5.4. In addition to the simpler model used in the previous works the electronic transfer at the electrode/film interface and the nanoporous nature of PB will be taken into account. Thanks to a more accurate experimental device and a fitting procedure to a more involved model, we were able to separate the kinetics of $\mathrm{K}^{+}$and $\mathrm{H}_{3} \mathrm{O}^{+}$cations in the charge compensation process.

\section{2 - MODEL}

In this part, we shall give a model of porous electroactive films which will be applied in the following to explain the PB film redox behaviour.

\section{1 - Generalities on porous electrodes}

In 1948 V. S. Daniel-Bekh and then in 1960 J. Euler and W. Nonnenmacher [20] have been the first to model porous electrodes. Later, a model based on a transmission line has been developed by de Levie $[21,22,23]$. The simplest models consider a cylindrical pore with length $L$ and radius $r_{0}$. The pore wall is supposed to react with the electrolytic solution. The behaviour of this pore called active differs from the pores called passive where reactions occur only at the pore end. The current flowing through the pore provokes an ohmic drop related to the resistivity of the electrolyte in the pore. So, as a part of the current passes through the pore wall, the local current varies with the pore depth $x$. This is the cause of the particular behaviour of porous electrodes: on the one hand, a potential and current distribution is observed in the pore and, on the other hand, an axial concentration distribution is also observed if reactions occur on the pore wall.

Therefore, the general modelling is based on three fundamental relationships which describe the electrode behaviour:

- Ohmic drop in the pore. At a distance $x$ from the pore entrance, an elementary ohmic drop can be considered:

$$
d e(x)=-\rho_{2} \cdot i(x) \cdot d x
$$

where $d e(x)(\mathrm{V})$ and $i(x)\left(\mathrm{A} . \mathrm{cm}^{-2}\right)$ are the potential and current at a distance $x$ from the pore entrance, $\rho_{2}(\Omega . \mathrm{cm})$ is the resistivity of the electrolyte in the pore and $d x$ is a distance increment.

- Interfacial kinetics on the pore wall At a distance $x$ from the pore entrance, a current, $\operatorname{di}(x)$ related to the electrochemical reaction on the pore wall can be written for a simple reaction: 


$$
d i(x)=-K \cdot c(x) \cdot d x
$$

where $\mathrm{K}$ is the rate constant which depends on the local potential $e(x)$ and $c(x)$ is the local concentration.

- Diffusion in the pore At a distance $x$ from the pore entrance, a variation of the active species concentration can be written following the Fick law:

$$
\frac{d c}{d t}=D \frac{\partial^{2} c}{d x^{2}}-K_{P} \cdot c(x)
$$

where $\mathrm{K}_{\mathrm{p}}$ is the rate constant related to a reaction in the volume of the pore.

However, to take into account the transport of the species is very complicated. In fact, this transport can be decomposed between an axial component which brings the reactive species from the «mouth» of the pore to the reaction zone and a radial component which goes from the pore axis to bring the species up to the pore wall where the reaction occurs. The transport was considered as radial by de Levie [21,22,23] or axial by Keddam et al. [24]. Usually, to take into account both ohmic drop and diffusion needs digital calculation. So, J. Newman and C. W. Tobias [25], C. Cachet and R. Wiart [26] and Paasch et al. [27] have given such a solution.

Another approach has been proposed recently by La Mantia et al. [28] where a coupling between the local potential and concentration is taken into account by assuming that the potential difference in the pore depends both on the ohmic drop (which depends on the local current density) and a diffusion ohmic drop (which depends on the concentration gradient).

Also, by considering porous films, Barker [29], Albery [30], Buck [31, 32] et Paasch $[33,34,35,36]$ have calculated the electrochemical impedance of an electrode/film/electrolyte system.

By assuming that the axial concentration gradient is negligible, i.e. $c(x)$ is a constant, R. de Levie has given the pore impedance, $Z_{P}(\omega)$, per pore cross section unit, under the form:

$$
Z_{P}(\omega)=\sqrt{\rho_{2} Z_{0}(\omega)} \operatorname{coth}\left[L \sqrt{\frac{\rho_{2}}{Z_{0}(\omega)}}\right]
$$

where $Z_{0}(\omega)$ is the faradaic impedance relative to the electrochemical reaction of the species at the electrolyte/pore wall which will be calculated below.

Then the penetration depth of the electrical perturbation signal can be defined such as

$$
\lambda=\sqrt{\frac{Z_{0}(\omega)}{\rho_{2}}}
$$


In the low frequency range $(\omega \rightarrow 0)$ and $Z_{0} » \rho_{2}$, (i. e. $L<<\lambda$, which means that the potential perturbation goes to the end of the pore), then $\operatorname{coth}\left[L \sqrt{\left.\frac{\rho_{2}}{Z_{0}(\omega)}\right]}\right]=\frac{1}{L \sqrt{\frac{\rho_{2}}{Z_{0}(\omega)}}}+\frac{L \sqrt{\frac{\rho_{2}}{Z_{0}(\omega)}}}{3}$, the pore impedance becomes $: Z_{P_{B F}}(\omega)=\frac{Z_{0}(\omega)}{L}+\frac{\rho_{2} L}{3}$. Hence, in the low frequency range, the impedance of a cylindrical pore is equal to that of the flat electrode of same area as the developed pore surface, but with a shift of the real part equal to the third of the ohmic drop of the pore $\Omega=\rho_{2} L$.

In the high frequency range $(\omega \rightarrow \infty)$ and $Z_{0} \ll \rho_{2}$, (i. e. $L>>\lambda$, which means that the potential perturbation does not practically enter the pore) then $\operatorname{coth}\left[L \sqrt{\frac{\rho_{2}}{Z_{0}(\omega)}}\right]=1$ therefore $Z_{p}(\omega)=\sqrt{\rho_{2} Z_{0}(\omega)}$ does not depend on the pore length. It is called the impedance of a semiinfinite cylindrical pore.

Therefore, impedance measurements allow the pore texture of a porous electrode (equivalent pore radius, $r_{0}$, and pore length, $L$ ) and the resistivity of the electrolyte in the pore to be characterized.

\section{2 - Local impedance due to the ionic transfer : $Z_{0}(\omega)$}

Ionic transfer occurs at the film/electrolyte interface [17,37,38] The flux of species $i$ ( $a$ : anion, $c$ : cation, $s$ : solvent), $J_{i}$, is considered as positive if the species is expulsed from the film:

$$
J_{i}>0 \text { pour } x>0
$$

If both cations, anions and solvent are involved in the charge compensation process, the associated mass variation, $\Delta m$ and the electric charge variation per surface unit, $\Delta q$, which flows through the electrode/film interface, are equal to:

$$
\begin{aligned}
& \Delta m=m_{c} \xi_{c}+m_{a} \xi_{a}+m_{s} \xi_{s} \\
& \Delta q=-F\left(\Delta \xi_{c}-\Delta \xi_{a}\right)
\end{aligned}
$$


where $m_{i}$ and $\xi_{i}$ are the molar mass and the quantity of moles of species $i$ exchanged, respectively.

The general doping mechanism of an electroactive film by a cation or an anion can be described by the following reactions:

$$
<P_{c}>+e^{-}+C^{+} \underset{k_{c}^{\prime}}{\stackrel{k_{c}}{\rightarrow}}<P_{c}, C^{+}>\quad \text { and } \quad<P_{a}>+A^{-} \underset{k_{a}}{\stackrel{k_{a}^{\prime}}{\rightarrow}}<P_{a}, A^{-}>+e^{-}
$$

where the $\left\langle P_{i}\right\rangle$ are free electroactive sites of the film available for cations $(i=c)$ and anions $(i=a),\left\langle P_{i}, C^{+}>\right.$, and $\left\langle P_{i}, A^{-}>\right.$the cations and anions inserted in the film associated to the sites.

Consequently, the instantaneous molar flux of species $i(c, a$, or $s)$ is $J_{i}=\frac{d \xi_{i}}{d t}$ and the concentration of species $i$ in thin films of thickness $d_{f}$ can be written under the form:

$$
\xi_{i}=d_{f} C_{i}
$$

By using the laws of the heterogeneous kinetics and reactions (10), the flux $J_{i}$ of cations and anions are:

$$
\begin{aligned}
& J_{a}\left(d_{f}\right)=-d_{f} \frac{d C_{a}}{d t}=k_{a}\left(C_{a}-C_{a \min }\right)-k_{a}^{\prime}\left(C_{a \max }-C_{a}\right) C_{a s o l} \\
& J_{c}\left(d_{f}\right)=-d_{f} \frac{d C_{c}}{d t}=k_{c}^{\prime}\left(C_{c}-C_{c \min }\right)-k_{c}\left(C_{c \max }-C_{c}\right) C_{c s o l}
\end{aligned}
$$

By using the Hillman solvation model [39] , the same relationship can be used for the solvent flux:

$$
J_{s}\left(d_{f}\right)=-d_{f} \frac{d C_{s}}{d t}=k_{s}^{\prime}\left(C_{s}-C_{s \min }\right)-k_{s}\left(C_{s \max }-C_{s}\right)
$$

where

- $\quad C_{i}$ is the concentration of species $i$ in the film, $C_{i s o l}$ is the concentration of species $i$ in the solution by assuming that the diffusion of the ions in solution does not limit the global kinetics;

- the term $\left[C_{\text {imax }}-C_{i}\right]$ is the concentration in free sites for species $i$ at time $t$, where $C_{\text {imax }}$ is the maximum concentration of the free sites in the film for species $i$;

- the term $\left[C_{i}-C_{\text {imin }}\right]$ is the concentration of species $i$ in the film bulk at time $t$, where $C_{\text {imin }}$ is the minimum concentration of species $i$ in the film.

Finally, the kinetic constants depend on potential under the form: 


$$
\begin{aligned}
& k_{i}^{\prime}=k_{i_{0}}^{\prime} e^{\left[b_{i}^{\prime}\left(E-E_{i}^{o}\right)\right]} \\
& k_{i}=k_{i_{o}} e^{\left[b_{i}\left(E-E_{i}^{o}\right)\right]}
\end{aligned}
$$

where $E$ is the potential, $E_{i}^{\circ}$ is the apparent normal potential, $\left(E-E_{i}{ }^{\circ}\right)$ is the overvoltage, $k_{i 0}$, $k_{i 0}^{\prime}, b_{i 0}$ and $b_{i 0}^{\prime}$ are constants.

\subsection{1 - Boundary limits}

By considering that the electrolyte in the pore contains only one type of cation and one type of anion and by using the Faraday law, the current density, $j_{F}$, at the film/electrolyte interface (at the pore wall which is characterized by an equivalent thickness $d_{f}$ )

$$
-J_{a}\left(d_{f}\right)+J_{c}\left(d_{f}\right)=\frac{j_{F}}{F}
$$

On the contrary, at the electrode/film interface $(x=0)$, where only electrons are exchanged, the flux of ions and solvent are:

$$
J_{a}=J_{c}=J_{s}=0
$$

Now, the insertion laws can be determined in steady and dynamic states.

\subsection{2 - Steady state}

At steady state, the system is at equilibrium and the flux $J_{i}$ are zero. Then, the steady state concentrations (insertion laws) are from equations (12-13-14).

$$
C_{i}(E)=\frac{C_{i \max } e^{\left[\left(b_{i}^{\prime}-b_{i}\right)\left(E-E_{i}^{o}-E_{i}\right)\right]}+C_{i \min }}{1+e^{\left[\left(b_{i}^{\prime}-b_{i}\right)\left(E-E_{i}^{o}-E_{i}\right)\right]}}
$$

by defining $E_{i}$ such as $C_{i s o l} \cdot \frac{k_{i 0}^{\prime}}{k_{i 0}}=e^{-\left(b_{i}-b_{i}^{\prime}\right) \cdot E_{i}}$.

\subsection{3 - Dynamic regime}

Under the effect of a potential sinusoidal perturbation with low amplitude, $\Delta E$, imposed to the electrode/film/electrolyte system, sinusoidal fluctuations of concentration, $\Delta C_{i}$, and flux, $\Delta J_{i}$, are observed such as:

$$
\Delta J_{i}=-d_{f} \frac{d C_{i}}{d t}=-j \omega d_{f} \Delta C_{i}
$$

On the other hand, the expression of the global insertion/expulsion flux, $\Delta J_{i}$, which depends on the concentration and potential perturbations, at the film/electrolyte interface is:

$$
\left.\left.\Delta J_{i}=\frac{\partial J_{i}}{\partial C_{i}}\right)_{E} \Delta C_{i}+\frac{\partial J_{i}}{\partial E}\right)_{C_{i}} \Delta E=K_{i} \Delta C_{i}\left(d_{f}\right)+G_{i} \Delta E
$$

where 


$$
\begin{gathered}
K_{a}=\left(\frac{\partial J_{a}}{\partial C_{a}}\right)_{E}=k_{a}+k^{\prime}{ }_{a} C_{a_{s o l}} \\
K_{c}=\left(\frac{\partial J_{c}}{\partial C_{c}}\right)_{E}=k_{c}^{\prime}+k_{c} C_{c_{s o l}} \\
G_{a}=\left(\frac{\partial J_{a}}{\partial E}\right)_{C_{a}}=\left[b_{a} k_{a}\left(C_{a}-C_{a_{\min }}\right)-b_{a}^{\prime} k_{a}^{\prime}\left(C_{a_{\max }}-C_{a}\right) C_{a_{s o l}}\right] \\
G_{c}=\left(\frac{\partial J_{c}}{\partial E}\right)_{C_{c}}=\left[b_{c}^{\prime} k_{c}^{\prime}\left(C_{c}-C_{c_{\min }}\right)-b_{c} k_{c}\left(C_{c_{\max }}-C_{c}\right) C_{c_{s o l}}\right]
\end{gathered}
$$

$K_{i}$ is the time constant of the kinetics of the species global transfer; $G_{i}$ is the inverse of the transfer resistance of the species at the film/solution interface (where $i$ is the cation $c$, the anion $a$ or the solvent $s$ ). From Equations (20-21) the insertion law can be written under the form:

$$
\frac{\Delta C_{i}}{\Delta E}(\omega)=\frac{-G_{i}}{j \omega d_{f}+K_{i}}
$$

which gives the concentration response to a potential perturbation.

\section{3 - Model of electroactive porous films with two inserted cations}

To study the behaviour of the electroactive porous films by ac-electrogravimetric technique, a model will be presented now. It is based on the insertion/expulsion of two different cations, $c_{1}$ and $c_{2}$, in independent sites in PB films, $P_{c_{1}}$ and $P_{c_{2}}$, such as:

$$
<P_{c_{1}}>+e^{-}+C_{1}^{+} \underset{k_{c_{1}}^{\prime}}{\stackrel{k_{c_{1}}}{\rightarrow}}<P_{c_{1}}, C_{1}^{+}>\quad<P_{c_{2}}>+e^{-}+C_{2}^{+} \underset{k_{c_{2}}^{\prime}}{\stackrel{k_{c_{2}}}{\rightarrow}}<P_{c_{2}}, C_{2}^{+}>
$$

To simplify the derivation, a few hypotheses are used:

$\checkmark$ As the electronic transfer is not infinitely fast, an electronic transfer resistance, $R_{e}$, is considered in parallel on a capacity across the electrode/film interface.

$\checkmark$ Ion transport in the solution and in the electroactive film is fast. 


\subsection{1 - Electrochemical impedance $\frac{\Delta E}{\Delta I}(\omega)$}

\subsubsection{1 - Faradaic impedance related to ionic transfer}

In dynamic regime, the perturbation $\Delta E$ leads to a variation of the charge in the film by considering that the two cations, $c_{1}$ and $c_{2}$, are involved in the charge compensation process using Equations (10). In addition, the faradaic current density is such that $\Delta I_{F}=j \omega \Delta q$. By using relationship (9) and by dividing by $\Delta E$, the Faradaic admittance is calculated for the insertion/expulsion of the two cations:

$$
\frac{\Delta I_{F}}{\Delta E}=-j \omega F d_{f}\left(\frac{\Delta C_{c_{1}}}{\Delta E}+\frac{\Delta C_{c_{2}}}{\Delta E}\right)
$$

Moreover, Equation (26) relative to the insertion law allows the impedance to be calculated:

$$
\frac{1}{Z_{F c_{i}}}=-j \omega F d_{f} \frac{\Delta C_{c_{i}}}{\Delta E}=j \omega F d_{f} \frac{G_{c_{i}}}{j \omega d_{f}+K_{c_{i}}}
$$

where $Z_{F i}$ is the Faradaic impedance relative to the ionic transfer of cation $i$, for which $G_{i}>0$

By using Equations (26 and 28), the faradaic impedance, $Z_{F}(\omega)$ relative to the global ionic transfer of the electroactive film for two species (cation and anion) involved in the charge compensation is:

$$
Z_{F}(\omega)=\frac{\Delta E}{\Delta I_{F}}(\omega)=\frac{1}{j \omega d_{f} F\left[\frac{G_{c_{1}}}{j \omega d_{f}+K_{c_{1}}}+\frac{G_{c_{2}}}{j \omega d_{f}+K_{c_{2}}}\right]}
$$

\subsubsection{2 - Electrochemical impedance of a porous electroactive film}

To simplify, the electroactive film is supposed to be constituted by cylindrical pores full of electrolyte where the insertion/expulsion reactions occurs on the wall (Figure 1).

Using the De Levie model given above, the impedance of the pore, $Z_{P}(\omega)$, is: [40]

where

$$
Z_{P}(\omega)=\sqrt{\rho_{2} Z_{0}(\omega)} \operatorname{coth}\left[L \sqrt{\frac{\rho_{2}}{Z_{0}(\omega)}}\right]
$$


is the Faradaic impedance per surface unit of pore wall, relative to the ionic transfer of species $Z_{F i}\left(i\right.$ : cations or anions) in parallel with a double layer capacity $C_{d}$ at the pore wall/electrolyte interface.

$$
Z_{0}(\omega)=\frac{1}{j \omega C_{d}+j \omega d_{f} F\left[\frac{G_{c_{1}}}{j \omega d_{f}+K_{c_{1}}}+\frac{G_{c_{2}}}{j \omega d_{f}+K_{c_{2}}}\right]}
$$

\subsubsection{3 - Global electrochemical impedance}

The global electrochemical impedance of the porous electroactive is the sum of the impedance related to the electronic transfer at the electrode/film interface (contact of PB with the metallic electrode), $Z_{e}$, the impedance of the pore, $Z_{P}$, and the solution resistance, $R_{s}$ :

$$
Z(\omega)=R_{s}+Z_{e}+Z_{P}=R_{s}+\frac{1}{\frac{1}{R_{e}}+j \omega C_{e}}+\sqrt{\rho_{2} Z_{0}(\omega)} \operatorname{coth}\left[L \sqrt{\frac{\rho_{2}}{Z_{0}(\omega)}}\right]
$$

where $R_{e}$ is the electronic transfer resistance and $C_{e}$ the capacity of the electrode/film interface.

At low frequency: $\omega \rightarrow 0, Z_{P}(\omega)=\frac{Z_{0}(\omega)}{L}+\frac{\rho_{2} L}{3}$,

and the global electrochemical impedance is then:

$$
Z_{B F}(\omega)=R_{s}+\frac{1}{\frac{1}{R_{e}}+j \omega C_{e}}+\frac{Z_{0}}{L}+\frac{\rho_{2} L}{3}
$$

\subsection{2 - Electrogravimetric transfer function $\frac{\Delta m}{\Delta E}(\omega)$}

The $\Delta E$ perturbation leads to a change of the charge compensation related to the species going in and out (cation, anion, and solvent) at the mouth of the pore. By considering that the mass response to the electrical sine wave perturbation occurs only in the low frequency range, the mass variation $\Delta m$, following Equation (8), dividing by $\Delta E$, and using relationship (11), the function $\frac{\Delta m}{\Delta E}(\omega)$ relative to the insertion/expulsion of the cations $c_{1}$ and $c_{2}$, and eventually solvent, becomes:

$$
\frac{\Delta m}{\Delta E}(\omega)=d_{f}\left[m_{c_{1}} \frac{\Delta C_{c_{1}}}{\Delta E}+m_{c_{2}} \frac{\Delta C_{c_{2}}}{\Delta E}+m_{s} \frac{\Delta C_{s}}{\Delta E}\right]
$$


In Equation (38), the global electrogravimetric transfer function per electrode surface unit taking into account all the involved species (cation, anion and solvent) is:

$$
\frac{\Delta m}{\Delta E}(\omega)=-d_{f}\left(m_{c_{1}} \frac{G_{c_{1}}}{j \omega d_{f}+K_{c_{1}}}+m_{c_{2}} \frac{G_{c_{2}}}{j \omega d_{f}+K_{c_{2}}}+m_{s} \frac{G_{s}}{j \omega d_{f}+K_{s}}\right)
$$

\section{$2.4 \quad$ - Experimental data treatment}

The experimental data were fitted to the model of the porous electroactive film described above to obtain the kinetic parameters by using a digital fitting procedure based on a Least Square Method (WNCLNSF software developed by J. Garcia-Jareno and F. Vicente, Univ. Valencia, Spain). The three transfer functions: electrochemical impedance, charge/potential transfer function, and the electrogravimetric transfer function were separately used.

\subsection{1 - Electrochemical impedance}

The fitting procedure applied to the electrochemical impedance in the frequency range $(0.01 \mathrm{~Hz}-60 \mathrm{kHz})$ and by considering only a global ionic transfer (where two cations are considered at the same time) with impedance $Z_{t}$, allows informations relative to the electronic and the global ionic transfer and the porosity of the electroactive film to be obtained. Diffusion is neglected as the film is very thin and typical responses, ie $45^{\circ}$ slopes, were not observed for the EIS responses.

By taking into account the electrolyte resistance, $R_{s}$, and by using Equation (35), the electrochemical impedance of the electroactive film is equal to:

$$
\begin{gathered}
\qquad Z(\omega)=R_{s}+Z_{p}+Z_{e} \\
\text { where } Z_{P}(\omega)=\sqrt{\rho_{2} Z_{0}(\omega)} \operatorname{coth}\left[L \sqrt{\frac{\rho_{2}}{Z_{0}(\omega)}}\right], Z_{0}(\omega)=\frac{1}{j \omega C_{d}+\frac{1}{Z_{t}}}, \\
\qquad Z_{e}(\omega)=\frac{1}{\frac{1}{R_{e}}+j \omega C_{e}} \text { and } Z_{t}(\omega)=R_{t}+\frac{1}{j \omega C_{t}}
\end{gathered}
$$

where $R_{t}$ is the ionic transfer resistance and $C_{t}$ is the global insertion capacity.

As $Z_{P}(\omega)$ can be also be written under the form:

$$
Z_{P}(\omega)=\sqrt{\rho_{2} L \frac{Z_{0}(\omega)}{L}} \operatorname{coth}\left[\sqrt{\frac{\rho_{2} L}{\frac{Z_{0}(\omega)}{L}}}\right]
$$


where $\frac{Z_{0}(\omega)}{L}=\frac{1}{j \omega C_{d} L+\frac{1}{\frac{Z_{F i}}{L}}}$

and $\frac{Z_{F i}}{L}=\frac{R_{t}}{L}+\frac{1}{j \omega C_{t} L}$

Therefore, all the quantities will be given per pore length unit.

Nine parameters $R_{s}, L, \rho_{2}, R_{i}, C_{i}, C_{d}, C_{e}, R_{e}$ (and $a_{m}$ when a CPE is considered relatively to $C_{d}$ ) are obtained thanks to the electrochemical impedance. The free parameters are $R_{s}, \rho_{2} L, R_{i} L, C_{i} L, C_{d} L, C_{e}, R_{e}$.

It is noticeable that for the electrochemical impedance, only one Faradaic impedance, $Z_{t}$, relative to the global ionic transfer which represents all the charged species participating to the charge compensation process is taken into account to simplify the fitting procedure. Thus two global quantities $R_{i}$ and $C_{i}$ are only obtained. The film response in the low frequency range, which is related to the ionic transfer, will be more precisely interpreted below by using the two other transfer functions. In this way, the kinetic constants $K_{i}$ and $G_{i}$ of the involved cations will be separately estimated.

\subsection{2 - Charge/potential transfer function}

The charge/potential transfer function, calculated from the experimental electrochemical impedance, is equal to:

$$
\frac{\Delta q}{\Delta E}(\omega)=\frac{1}{j \omega} \frac{1}{Z_{0}}
$$

gives information on the ionic transfer. For the fitting, it can be conveniently written under the form:

$$
\frac{\Delta q}{\Delta E}(\omega)=C_{d}+\frac{1}{j \omega} \sum_{i}\left(\frac{1}{R_{i}+\frac{1}{j \omega C_{i}}}\right)
$$

By using Equation (31),

$$
\frac{1}{Z_{F i}}=j \omega \cdot F \cdot d_{f} \frac{G_{i}}{j \omega d_{f}+K_{i}},
$$

the relationships between $R_{i}, C_{i}$ and $G_{i}, K_{i}$ can be deduced for all the involved species: 


$$
\begin{aligned}
Z_{F i} & =R_{i}+\frac{1}{j \omega C_{i}}=\frac{1}{F G_{i}}\left(1+\frac{K_{i}}{j \omega d_{f}}\right) \\
\text { therefore } \quad G_{i} & =\frac{1}{F R_{i}} \quad \text { and } \quad K_{i}=d_{f} \frac{1}{R_{i} C_{i}}
\end{aligned}
$$

For each charged species, three parameters $R_{i}, C_{i}$ (and $a_{f}$ when the insertion capacity is considered as a CPE) have to be fitted by considering only the low frequency range $(0.01 \mathrm{~Hz}$ $-100 \mathrm{~Hz}$ ) where $\frac{\Delta q}{\Delta E}$ and $\frac{\Delta m}{\Delta E}$ are of interest (which correspond to the quasi-vertical capacitive part of the electrochemical impedance). As soon as these three parameters are determined, they are used for the fitting of the electrogravimetric transfer function.

\subsection{3 - Electrogravimetric transfer function}

For each species, four parameters $R_{i}, C_{i}$, $a_{f}$ et $m_{i}$ have to be determined. Equation (39) is used for the fitting in the same frequency range of $\frac{\Delta q}{\Delta E}$. Finally, the kinetic parameters $K_{i}$ and $G_{i}$ are determined from the components $R_{\mathrm{i}}$ and $C_{\mathrm{i}}$ of the equivalent circuit.

$$
\frac{\Delta m}{\Delta E}(\omega)=\sum \frac{m_{i}}{F} \frac{1}{j \omega Z_{i}}=L \sum_{i} \frac{m_{i}}{F} \frac{1}{j \omega\left(R_{i}+\frac{1}{j \omega C_{i}}\right)}=d_{f} \sum_{i} m_{i} \frac{G_{i}}{j \omega d_{f}+K_{i}}
$$

In all these fitting procedures, the thickness of the film was taken equal to $100 \mathrm{~nm}$ as determined experimentally (see below).

\section{3 - EXPERIMENTAL}

The PB films were obtained by electrodeposit, in galvanostatic mode, on the gold electrode of a quartz resonator (AWS Company, Spain) acting as a classical working electrode, from a $0.02 \mathrm{M} \mathrm{K}_{3}\left(\mathrm{Fe}(\mathrm{CN})_{6}\right.$ ) (Prolabo-93.3\%), 0.02M FeCl 3 (Prolabo-99.4\%) and 0.01M HCl (Prolabo-37\%) solution. A $40 \mu \mathrm{A} \mathrm{cm} \mathrm{cm}^{-2}$ cathodic current was applied during $150 \mathrm{~s}$, to obtain a 100 $\mathrm{nm}$ thick PB film. The film thickness was estimated by SEM measurements. The film was then cycled 15 times in aqueous medium containing $0.5 \mathrm{M} \mathrm{KCl}$ (Prolabo-99.7\%) pH 2.5 obtained by adding $\mathrm{HCl}$. This medium was chosen to have a better stability of the films. Finally, the electrochemical studies were carried out in the same medium.

The film behaviour was investigated first by cyclic electrogravimetry and then by $a c$ electrogravimetry. $A c$-electrogravimetric measurements were carried out by using a 4 channels 
frequency response analyser (Solartron 1254 TFA) and home-made potentiostat and quartz crystal microbalance. Details of the experimental equipment are given elsewhere [17, 41, 42] Films are considered as acoustically thin enough and this hypothesis is fully justified with PB film thickness in the sub-micrometric range [43]

The PB films were tested in various conditions in aqueous media, $0.5 \mathrm{M} \mathrm{KCl}$ at $\mathrm{pH} 2.5$,

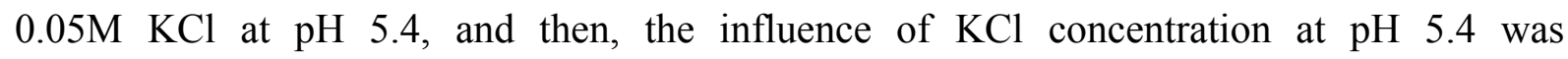
investigated.

\section{4 - RESULTS}

\section{1- Investigation of PB films in $0.5 \mathrm{M} \mathrm{KCl}$ at pH 2.5}

\subsection{1. - Cyclic electrogravimetry}

The PB film behaviour was studied in the potential domain where the PB↔ES conversion occurs (Equation 1). Figure 2 presents the changes of mass and current in a $-0.2 \mathrm{~V}$ à $0.6 \mathrm{~V}$ vs SCE (Saturated Calomel Electrode) potential range obtained at the $15^{\text {th }}$ cycle for a $\mathrm{PB}$ film immersed in $0.5 \mathrm{M} \mathrm{KCl}$ at $\mathrm{pH}$ 2.5. So, the electronic charge transfer at the film/electrode interface (change of the current) can be correlated to the ionic transfer at the film/electrolyte interface (change of the mass). The mass increased during the reduction and decreased during the oxidation. This confirms that the film electroneutrality was maintained by cation participation as it has been already shown that $\mathrm{K}^{+}$ions mainly participate to the charge compensation in the negative potentials range whereas protons, $\mathrm{H}_{3} \mathrm{O}^{+}$and $\mathrm{H}^{+}$, are mainly involved towards the positive potentials [44].

\subsection{2- Study of the PB film at 0.175 V vs SCE}

$A c$-electrogravimetric measurements on a $\mathrm{PB}$ film were carried out in $0.5 \mathrm{M} \mathrm{KCl}-\mathrm{pH}$ 2.5 at various potentials. At each imposed potential, the mass, $\Delta m(\omega)$, and the current, $\Delta I(\omega)$, responses of the film to a potential sinusoidal perturbation of low amplitude $\Delta E(\omega)(50 \mathrm{mV})$ were measured at various modulation frequencies between $63 \mathrm{kHz}$ and $10 \mathrm{mHz}$ by using a four channels frequency response analyser. This value of amplitude was selected as it is a good trade-off between linearity and accuracy.

As an example, the full data processing of the experimental data obtained on the PB film polarized at $0.175 \mathrm{~V}$ vs SCE is first given. Figure 3 shows the three principal transfer functions, two experimental $\frac{\Delta E}{\Delta I}(\omega)$ and $\frac{\Delta m}{\Delta E}(\omega)$ and another one calculated $\frac{\Delta q}{\Delta E}(\omega)$, of a PB film at $0.175 \mathrm{~V}$ vs. SCE. Based on the model described above, the three transfer functions 
were separately fitted under WINCLNSF and the estimated parameters are given in Figure $2 \mathrm{~d}$. The electrochemical impedance $\frac{\Delta E}{\Delta I}(\omega)$ (Figure 3a) had the classical shape of the behaviour of a blocked electrode with a quasi-vertical capacitive part in the low frequency range. By considering a global ionic transfer, the electronic transfer resistance, $R_{e}=0.89 \Omega$ was shown to be lower than the global ionic transfer resistance, $R_{t}=22 \Omega$. This demonstrates that ionic transfer at the film/electrolyte interface limits the kinetics of the film reduction or oxidation. At last, a value of $1.5 \Omega \mathrm{cm}$ was obtained for the pore resistivity of the electrolyte in the film. In addition, this result shows that electronic transfer is fast as it responds in the high frequency range, between $63 \mathrm{kHz}$ and $1 \mathrm{kHz}$. Ionic transfer is slower as it responds in the low frequency range and it occurs at the pore surface with a potential distribution along the pore.

The second transfer function, $\frac{\Delta q}{\Delta E}(\omega)$, which is calculated by using the experimental data of the electrochemical impedance (eq. 42), is shown in Figure 3b. The two, not well separated, loops displayed at this potential pointed out that there are two charged species involved in the film redox process. This can be either two cations or two anions or one cation and one anion. A fitting procedure based on Equation (43) allowed the ionic transfer resistance and the insertion capacity of each ion to be estimated and then the kinetic parameters $K_{i}$ and $G_{i}$ (Figure $3 \mathrm{~d}$ ).

The electrogravimetric transfer function, $\frac{\Delta m}{\Delta E}(\omega)$, also displays two loops (Figure 3c). They are located in the third quadrant which corresponds to the expulsion of two cations during the film oxidation. After the fitting procedure based on Equation (47) the atomic masses of the involved cations are determined, their values show that the two detected cations are the potassium $\left(m_{c l}=39\right.$ g.mole $\left.{ }^{-1}\right)$ related to the slowest process $\left(K_{c_{1}}\right)$ and the hydronium $\left(m_{c 2}=19 \mathrm{~g} \mathrm{~mole}^{-1}\right)$ related to the fastest process $\left(K_{c_{2}}\right)$. This means that the $\mathrm{H}_{3} \mathrm{O}^{+}$transfer kinetics is faster than the $\mathrm{K}^{+}$transfer. The two cations participate in the same direction to the charge compensation. They are expulsed towards the electrolyte during the film oxidation and are inserted in the film from the electrolyte during the film reduction with well-defined kinetics for each ion. It is the first time that the separate contribution of the two involved species is demonstrated and that their kinetics are clearly determined.

\subsection{3 - Study of the PB film behaviour with respect to the potential}


In a similar way, fittings were carried out for all the analysed potentials between 0 and $0.325 \mathrm{~V}$ vs. SCE. Figure $4 \mathrm{a}$ shows the variation of the electronic transfer resistance, $R_{e}$, and of the ionic transfer resistance, $R_{t}$, obtained from the electrochemical impedance at each potential. They show a minimum at the potentials where the PB↔ES conversion occurs. In addition, the ionic transfer resistance is always higher than the electronic transfer resistance whatever the imposed potential to the PB film. This means that the kinetics of the global charge transfer of the PB film is controlled by ionic transfer during a redox process whatever the potential. However, the ionic transfer resistance, $R_{t}$, obtained from the impedance is a global resistance for all the charged species involved in the electrochemical process. Moreover, the pore resistivity, $\rho_{2}$, changes with respect to the potential (Figure $4 \mathrm{~b}$ ) with a minimum, in the vicinity of the $\mathrm{PB} \leftrightarrow \mathrm{ES}$ conversion potential, which could have two origins. On the one hand, the impoverishment of cations in the pore when going towards negative potentials, where cations enter the film, leads to an increase of the resistivity. On the other hand, the change of the zeolitic structure related to the lattice contraction when the film is oxidized induce an increase of the pore resistivity due to the decrease of the pore diameter. ${ }^{45}$, 46

The $K_{c}$ and $G_{c}$ kinetic constants, are plotted on Figure 4 with respect to the potential. $G_{c}$ is greater for $\mathrm{K}^{+}$than $\mathrm{H}_{3} \mathrm{O}^{+}$for whatever the imposed potential. In addition, the quantity $d C_{c} / d E$, which is the derivative of the concentration with respect to the imposed potential, for each species, can be calculated by considering the low frequency limit of $\frac{\Delta C_{i}}{\Delta E}(\omega)$, then:

$$
\frac{\Delta C_{i}}{\Delta E}(\omega)=\frac{-G_{i}}{j \omega d_{f}+K_{i}} \rightarrow \frac{\Delta C_{i}}{\Delta E}(0)=-\frac{G_{i}}{K_{i}}
$$

The variation of this quantity for the two cations $\mathrm{K}^{+}$and $\mathrm{H}_{3} \mathrm{O}^{+}$, is shown on Figure $4 \mathrm{e}$ with respect to the potential. $d C_{c} / d E$ reaches a maximum at the potential of the $\mathrm{PB} \leftrightarrow \mathrm{ES}$ conversion. This corresponds to the maximum of the current of the cyclic voltammogram (Figure 2).

The change of the relative concentration in the film of each cation can be calculated with respect to the potential by integrating the quantity $d C_{c} / d E$. Figure $4 \mathrm{f}$ shows that the relative concentration of the species in the film decreases when the potential becomes more anodic. The negative sign means that the cation is expulsed from the film during its oxidation. The $\mathrm{K}^{+}$concentration change between the two states, reduced and oxidized, $\left(\Delta C_{c l}=3.3\right.$ mmole. $\left.\mathrm{cm}^{-3}\right)$ is greater than the concentration change of $\mathrm{H}_{3} \mathrm{O}^{+}\left(\Delta C_{c 2}=1.4\right.$ mmole. $\left.\mathrm{cm}^{-3}\right)$. So, 
$\mathrm{H}_{3} \mathrm{O}^{+}$contributes by $33.6 \%$ to the total flux of the species exchanged between 0 and $0.35 \mathrm{~V}$ vs. SCE. This demonstrates that $\mathrm{K}^{+}$plays a greater role than $\mathrm{H}_{3} \mathrm{O}^{+}$in the charge compensation process.

\section{2 - Investigation of PB films in $0.05 \mathrm{M} \mathrm{KCl} 0.05 \mathrm{M}$ at $\mathrm{pH} 5.4$}

\subsection{1 - Cyclic electrogravimetry}

When the film was converted under the soluble form by applying 15 consecutive cycles in $0.5 \mathrm{M} \mathrm{KCl}-\mathrm{pH} 2.5$, the investigation of the redox properties of the PB film was carried out in a $0.05 \mathrm{M} \mathrm{KCl}-\mathrm{pH} 5.4$ aqueous medium.

Figure 5 shows the shapes of the current-potential and mass-potential curves. They are similar as those plotted in the $0.5 \mathrm{M} \mathrm{KCl}-\mathrm{pH} 2.5$ aqueous medium (Figure 2). However, the redox peak occurs at a more cathodic potential, around 0.1V vs. SCE. The charges estimated between the two $\mathrm{pH}$ values are in the same order of magnitude as mainly related to the electronic transfer and indirectly connected to the ionic transfers in term of kinetics. In addition, the mass change between the reduced and oxidized states is smaller $\left(1.5 \mu \mathrm{g} \mathrm{cm}^{-2}\right)$ than at $\mathrm{pH} 2.5$ and the sense of variation shows that, like at $\mathrm{pH} 2.5$, the reversible $\mathrm{PB} \leftrightarrow \mathrm{ES}$ conversion is mainly due to cations transfer.

\subsection{2 - Ac-Electrogravimetry}

As previously, the three transfer functions were obtained for the PB film in $0.05 \mathrm{M}$ $\mathrm{KCl}$ at $\mathrm{pH} 5.4$ between $-0.1 \mathrm{~V}$ and $0.35 \mathrm{~V}$ vs SCE. Then, the fitting procedure was used.

The shapes of the three transfer functions were very close to those obtained at $\mathrm{pH} 2.5$. By using the model of the porous electroactive film given previously, the fitting procedure was applied separately on each transfer function for all potentials. With this model, it is noticeable that a good agreement can be found between the experimental and the calculated diagrams. The electrochemical impedance has still a shape similar to the one of a blocking electrode. The charge/potential transfer function presents two loops corresponding to the relaxations of the two cations $\mathrm{K}^{+}$and $\mathrm{H}_{3} \mathrm{O}^{+}$. The electrogravimetric transfer function shows the presence of a third species.

The analysis of the electrochemical impedance gives information on the charge transfer of the PB film during the electrochemical process. Like at $\mathrm{pH} 2.5$, the rate limiting step of the global charge transfer is the ionic transfer at the film/electrolyte interface as the value of the ionic transfer resistance, $R_{t}$, is always larger than the resistance corresponding to 
the electronic transfer, $R_{e}$, whatever the potential applied to the PB film (Figure 6a). However, at $\mathrm{pH} 5.4$, the process is slower as the resistances $R_{t}$ and $R_{e}$ determined for $0.05 \mathrm{M} \mathrm{KCl}$ at $\mathrm{pH}$ 5.4 are much larger than those determined for $0.5 \mathrm{M} \mathrm{KCl}$ at $\mathrm{pH} 2.5$ (Figure 4a). This can be explained by the decrease of both the $\mathrm{KCl}$ and protons concentration for $0.05 \mathrm{M} \mathrm{KCl}$ at $\mathrm{pH}$ 5.4. The resistivity of the pore (Figure $6 \mathrm{~b}$ ) also changes with both concentrations and potential and it is higher for $0.05 \mathrm{M} \mathrm{KCl}$ at $\mathrm{pH} 5.4$. This is mainly due to a higher resistivity of the solution at pH $5.4\left(R_{s}=32 \Omega\right)$ than at $\mathrm{pH} 2.5\left(R_{s}=3.8 \Omega\right)$.

The fitting of the experimental electrogravimetric transfer function $\frac{\Delta m}{\Delta E}(\omega)$ allows the values of the kinetic constants $K_{c}$ and $G_{c}$ to be determined. The estimated atomic masses $m_{c_{1}}=39 \mathrm{~g} \mathrm{~mole}^{-1}, m_{c_{2}}=19 \mathrm{~g} \mathrm{~mole}^{-1}$ and $m_{s}=18 \mathrm{~g} \mathrm{~mole}^{-1}$ show that the third involved species is the solvent. These quantities are plotted with respect to the potential in Figure $6 \mathrm{c}$ and d. Like at $\mathrm{pH} 2.5, \mathrm{H}_{3} \mathrm{O}^{+}$insertion is the fastest process as the quantities $K_{c}$, which is the time constant of the cation movement is larger for $\mathrm{H}_{3} \mathrm{O}^{+}$than for $\mathrm{K}^{+}$and the solvent.. Nevertheless, the constants $G_{c}$ for $\mathrm{K}^{+}$and $\mathrm{H}_{3} \mathrm{O}^{+}$are smaller for $0.05 \mathrm{M} \mathrm{KCl}$ at $\mathrm{pH} 5.4$. Moreover, the fitting for the electrochemical impedance points out that the global ionic transfer resistance, $R_{t}$, of the two cations $\mathrm{K}^{+}$and $\mathrm{H}^{+}$is larger at $\mathrm{pH} 5.4$, which leads to an average constant $G_{t}$ smaller than at $\mathrm{pH} 2.5$ from the relationship $G_{t}=1 / 96500 R_{t}$. Consequently, the constants $K_{c}$ and $G_{c}$ associated to the $\mathrm{K}^{+}$and $\mathrm{H}_{3} \mathrm{O}^{+}$ions are smaller and are correlated to the $\mathrm{K}^{+}$and $\mathrm{H}^{+}$concentrations.

The change of the derivatives of the insertion law for the $\mathrm{K}^{+}$and $\mathrm{H}_{3} \mathrm{O}^{+}$ions are given in Figure 6e with respect to the potential. The value of $d C_{c} / d E$ reaches a maximum at the potential where the PB↔ES conversion occurs. This is in good agreement with the cyclic electrogravimetric curve (Figure 5). The ratio between the two values of $d C_{c} / d E$ for $\mathrm{K}^{+}$and $\mathrm{H}_{3} \mathrm{O}^{+}$is larger at the negative potentials between $0.0 \mathrm{~V}$ and $0.2 \mathrm{~V}$ (Figure 6e). This means that the role of the $\mathrm{K}^{+}$ion is predominant at negative potentials whereas the role of $\mathrm{H}_{3} \mathrm{O}^{+}$is predominant at positive potentials. Nevertheless, the same conclusion was reached when only one global insertion rate, $K_{c}$, was considered for an apparent average mass of $\mathrm{K}^{+}$and $\mathrm{H}_{3} \mathrm{O}^{+}[17]$ as the apparent mass of the positive species is larger at negative potentials than at positive potentials.

The change of the concentration, in the PB film between the reduced and oxidized states, of $\mathrm{K}^{+}\left(\Delta \mathrm{C}_{\mathrm{c} 1}=3.4\right.$ mmole $\left.\mathrm{cm}^{-3}\right)$ is larger than the one for $\mathrm{H}_{3} \mathrm{O}^{+}\left(\Delta \mathrm{C}_{\mathrm{c} 2}=0.7\right.$ mmole $\mathrm{cm}^{-}$ ${ }^{3}$ ). So, $17.0 \%$ of $\mathrm{H}_{3} \mathrm{O}^{+}$contributes to the total flux of the exchanged species between -0.05 
and $0.35 \mathrm{~V}$ vs. SCE. This means that the $\mathrm{K}^{+}$ion plays a more important role than the $\mathrm{H}_{3} \mathrm{O}^{+}$ion in the charge compensation during the redox switching of the PB film. As there is less protons at $\mathrm{pH} 5.4$ than at $\mathrm{pH} 2.5$, it is normal that the quantity of implied $\mathrm{H}_{3} \mathrm{O}^{+}$ions is lower at $\mathrm{pH}$ 5.4 .

\section{3 - Influence of the $\mathrm{KCl}$ concentration at $\mathrm{pH} 5.4$}

The influence of the $\mathrm{KCl}$ concentration in aqueous solutions at $\mathrm{pH} 5.4$ on the electrogravimetric response of a PB film was studied. The soluble PB film was preconditioned in $0.1 \mathrm{M} \mathrm{KCl}$ during one hour. The electrochemical measurements were carried out on the same film by increasing the $\mathrm{KCl}$ concentration from $0.01 \mathrm{M}$ to $0.5 \mathrm{M}$. At each concentration, the cyclic electrogravimetric measurement was carried out during three cycles in order to obtain a well equilibrated film before the ac-electrogravimetric measurements carried out at a potential $0.25 \mathrm{~V}$ vs. SCE.

\subsection{1 - Cyclic electrogravimetry}

The current-voltage curves are given in Figure 7a for various $\mathrm{KCl}$ concentrations. The anodic redox peak related to the $\mathrm{PB} \leftrightarrow \mathrm{ES}$ conversion shifts towards more positive potentials when the $\mathrm{KCl}$ concentration increases. The change of the potential of the cathodic peak is more important than the change of the anodic peak when the $\mathrm{KCl}$ concentration varies from $0.01 \mathrm{M}$ to $0.5 \mathrm{M}$. This dissymmetry of the current responses to the cathodic and anodic potential sweeps can be explained by considering that cationic transfer occurs on the one hand from the film, where the cations concentration is independent of the solution, for the anodic sweep and on the other hand from the solution, where cation concentration changes, towards the film, for the cathodic sweep. For this reason, the current response is more influenced by the cation concentration in the solution when the potential decreases.

In addition, the electrochemical processes are more reversible at high concentration because the two potentials of the anodic $\left(E_{a}\right)$ and cathodic $\left(E_{c}\right)$ peaks are closer (Table 1). This indicates also that ionic transfer at the film/solution interface is probably the major limiting process for the redox switching of the $\mathrm{PB}$ film in $\mathrm{KCl}$ aqueous media compared to electronic transfer at the electrode/film interface.

The two potentials of the anodic and cathodic peaks allow the formal potential $\left(E_{p-p}\right)$ to be calculated at each concentration (Table 1) following the equation: 


$$
E_{p-p}=\frac{E_{a}+E_{c}}{2}
$$

The linear fitting of the formal potential, $E_{p-p}$, with respect to the logarithm of the $\mathrm{KCl}$ concentration gives a value of $43 \mathrm{mV} /$ decade of $\mathrm{KCl}$ concentration. This value is rather far from the theoretical value $(59 \mathrm{mV} /$ decade) given by the Nernst equation. If the redox process is supposed to be due to the potassium participation, the value $43 \mathrm{mV} /$ decade corresponds to the $\mathrm{PB} \leftrightarrow \mathrm{ES}$ reaction with a $\mathrm{K}^{+}$cation for a reduced $\mathrm{Fe}^{3+}$ ion.

From the $i-E$, curve, the anodic $\left(Q_{a}\right)$ and cathodic $\left(Q_{c}\right)$ charges were determined as well (Table 1). In spite of the fact that the anodic and cathodic peaks are not at the same potential, the anodic and cathodic charges are almost equal at each concentration. This means that the redox process related to the $\mathrm{PB} \leftrightarrow \mathrm{ES}$ reaction is reversible whatever the studied concentration.

The mass differences between the two potentials $-0.2 \mathrm{~V}$ and $0.6 \mathrm{~V}$ vs. SCE (Figure $7 \mathrm{~b}$ ) are practically equal $\left(1.3 \mu \mathrm{g} . \mathrm{cm}^{-2}\right)$ whatever the $\mathrm{KCl}$ concentration and the direction of the potential sweeping. This mass difference is proportional to the charge by assuming that the reaction process is only due to the cations participation $\left(\mathrm{K}^{+}\right.$and $\left.\mathrm{H}_{3} \mathrm{O}^{+}\right)$. This confirms the hypothesis of a reversible reaction process in the $-0.2 \mathrm{~V}$ and $0.6 \mathrm{~V}$ potential range.

However, the slope of the $\Delta m-E$ curves changes with the concentration. From the $\Delta m-$ $E$ curve plotted at each concentration, the slope of the cyclic $\Delta m / \Delta E$ can be estimated and will be compared below with the low frequency value of $\Delta m / \Delta E(\omega)$ obtained from the $a c$ electrogravimetric experiments. In fact, in the zone between 0.2 and $0.3 \mathrm{~V}$ vs. SCE, the change of mass is practically linear, in the two directions, anodic, $\Delta m / \Delta E_{a}$, and cathodic, $\Delta m / \Delta E_{c}$ (Table 1). However, a discrepancy can be observed between the cathodic and anodic values at each concentration. This dissymmetry is due to the fact that the PB film response is more influenced by the $\mathrm{KCl}$ concentration in the reduction direction than in the oxidation direction. However, these values increase with the $\mathrm{KCl}$ concentration whatever the direction of the sweeping potential.

\subsection{2 - Ac-electrogravimetry}

\subsubsection{1 - Electrochemical impedance}

The response of the electrochemical impedance of the PB film at $0.25 \mathrm{~V}$ vs. SCE at various $\mathrm{KCl}$ concentrations is shown in Figure 8a. First, the size of the high frequency capacitive loop decreases when the $\mathrm{KCl}$ concentration increases. This implies that ionic 
transfer is facilitated. The fitting procedure allows the various parameters which characterize the global charge transfer of the film to be calculated. The two transfer resistances $R_{e}$ and $R_{t}$ decrease when the $\mathrm{KCl}$ concentration increases (Figure $8 \mathrm{~b}$ ). This demonstrates an acceleration of the processes when the $\mathrm{KCl}$ concentration increases. In addition, the global charge transfer process is always controlled by ionic transfer as the ionic resistance is always larger than the electronic resistance whatever the studied $\mathrm{KCl}$ concentration (Figure 8c). However, the ratio between $R_{t}$ and $R_{e}$ decreases when the $\mathrm{KCl}$ concentration increases. This means that, when $\mathrm{KCl}$ concentration increases, as ionic transfer is accelerated the global charge transfer is more and more controlled by electronic transfer.

The fitting of the electrochemical impedance diagram also allows the pore resistivity, $\rho_{2}$, to be calculated (Figure $8 \mathrm{~d}$ ). This quantity decreases when the $\mathrm{KCl}$ concentration increases, due to the increase of the conductivity. Hence, it is correlated with the electrolyte conductivity characterized by $R_{s}$.

\subsubsection{2 - Mass-potential transfer function}

Figure 9a compares the electrogravimetric transfer functions, $\Delta m / \Delta E(\omega)$, for a $\mathrm{PB}$ film polarized at $0.25 \mathrm{~V}$ vs. $\mathrm{SCE}$ at various $\mathrm{KCl}$ concentrations. The loops, which are located in the third quadrant, correspond predominantly to cation insertion. In fact, the increase of the $\mathrm{KCl}$ concentration favours the species insertion at the film/solution interface and consequently, the size of the $\Delta m / \Delta E(\omega)$ loop increases with the $\mathrm{KCl}$ concentration. The fittings were carried out as previously by considering the two cations $\mathrm{K}^{+}$and $\mathrm{H}_{3} \mathrm{O}^{+}$and the solvent.

It is noticeable that the point measured at $0.025 \mathrm{~Hz}$ of the electrogravimetric curves in the aqueous solution at $\mathrm{pH}=5.4$ corresponds roughly to a $20 \mathrm{mV}^{-1} \mathrm{~s}^{-1}$ scan rate on a $0.8 \mathrm{~V}$ sweeping range in cyclic electrogravimetry. It should be point out that the role of $\mathrm{H}_{3} \mathrm{O}^{+}$is preponderant at this frequency.

The $\left|\frac{\Delta m}{\Delta E}\right|$ values extracted from the ac-electrogravimetric curves increase with the $\mathrm{KCl}$ concentration (Table 2). Additionally, by comparing with the calculated values obtained from the cyclic electrogravimetry they are close to the anodic values for concentrations between $0.15 \mathrm{M}$ and $0.5 \mathrm{M}$ whereas for more diluted concentrations, between $0.01 \mathrm{M}$ and $0.1 \mathrm{M}$, they are close to the cathodic values. 
Figure $9 \mathrm{~b}$ and c show the variations of $K_{c}$ and $G_{c}$ for $\mathrm{K}^{+}, \mathrm{H}_{3} \mathrm{O}^{+}$, and the solvent with respect to the $\mathrm{KCl}$ concentration. As the studied media have a $\mathrm{pH}$ equal to 5.4 , the proton concentration does not change with the increase of the $\mathrm{KCl}$ concentration. The kinetic constant $K_{c}$ of the inserted species do not change very much with the $\mathrm{KCl}$ concentration. These results were obtained at $0.25 \mathrm{~V}$ vs. SCE where the $\mathrm{PB}$ film rather favours $\mathrm{H}_{3} \mathrm{O}^{+}$ insertion compared with the negative potentials (0-0.2V vs. SCE) $[17,44]$.

The kinetic constant $G_{i}$ for $\mathrm{H}_{3} \mathrm{O}^{+}$and the solvent increase and then become constant with respect to the potential contrarily to the one of $\mathrm{K}^{+}$which increases continuously with $\mathrm{KCl}$ concentration.

The values of $d C_{c} / d E$ were also calculated for the three species $\mathrm{K}^{+}, \mathrm{H}_{3} \mathrm{O}^{+}$and the solvent. The same evolution of this quantity can be observed for the two cations. The potassium plays a more important role than the hydronium in the charge compensation process because the value of $d C_{i} / d E$ is larger for $\mathrm{K}^{+}$than for $\mathrm{H}_{3} \mathrm{O}^{+}$. In addition, the ratio $\left(d C_{\mathrm{H}_{3} \mathrm{O}^{+}} / d E\right) /\left(d C_{K^{+}} / d E\right)$ decreases with the $\mathrm{KCl}$ concentrations (Figure 9d). This means that the role of $\mathrm{H}_{3} \mathrm{O}^{+}$is less and less important for the large $\mathrm{KCl}$ concentrations as $d C_{c} / d E$ is related to the facilities of the ion insertions. At this potential, the process is more and more limited by the kinetics of the potassium ions.

\section{5 - DISCUSSION}

The kinetics of the electrochemical process of the $\mathrm{PB}$ film in $\mathrm{KCl}$ media is mainly controlled by ionic transfer at the film/solution interface. It is mainly related to the participation of two cations, $\mathrm{K}^{+}$and $\mathrm{H}_{3} \mathrm{O}^{+}$, which was already suggested, especially at low $\mathrm{pH}$, for the reaction mechanisms in the literature $[12,16,47]$ The soluble and hydrated PB films contain about 14 to 16 water molecules for a mesh unit. Although water movement is clearly detected only for $\mathrm{pH}$ 5.4, the water located in the interstitial sites of the hydrated structure of $\mathrm{PB}$ can play a very important role for the cations $\mathrm{K}^{+}$and $\mathrm{H}_{3} \mathrm{O}^{+}$movement whatever the $\mathrm{pH}$ value. Gimenez-Romero et al. [48] have proposed the following mechanism for the insertion reaction of the cations $\mathrm{K}^{+}$and $\mathrm{H}_{3} \mathrm{O}^{+}$in a $\mathrm{PB}$ film, $\mathrm{K}_{h} \mathrm{Fe}_{k}^{3+}\left[\mathrm{Fe}^{2+}(\mathrm{CN})_{6}\right] . m \mathrm{H}_{2} \mathrm{O}$ by considering the dual insertion mechanism:

$$
\begin{aligned}
& K_{h} \mathrm{Fe}_{k}^{3+}\left[\mathrm{Fe}^{2+}(\mathrm{CN})_{6}\right]_{l} \cdot \mathrm{mH}_{2} \mathrm{O}+n \mathrm{~K}_{\text {solution }}^{+}+n e^{-} \stackrel{ }{\leftarrow} \\
& K_{h+n} \mathrm{Fe}_{k-n}^{3+} \mathrm{Fe}_{n}^{2+}\left[\mathrm{Fe}^{2+}(\mathrm{CN})_{6}\right\rfloor_{l} \cdot m \mathrm{H}_{2} \mathrm{O}
\end{aligned}
$$




$$
\begin{aligned}
& \mathrm{K}_{h} \mathrm{Fe}_{k}^{3+}\left[\mathrm{Fe}^{2+}(\mathrm{CN})_{6}\right], \mathrm{mH}_{2} \mathrm{O}+n \mathrm{H}_{3} \mathrm{O}_{\text {solution }}^{+}+n e^{-\stackrel{\leftarrow}{\leftarrow}} \\
& \mathrm{K}_{h} \mathrm{Fe}_{k-n}^{3+} \mathrm{Fe}_{n}^{2+}\left[\mathrm{Fe}^{2+}(\mathrm{CN})_{6} \mid, \mathrm{mH}_{2} \mathrm{O}\left(\mathrm{nH}_{3} \mathrm{O}^{+}\right)\right.
\end{aligned}
$$

The authors have suggested that the hydrated PB film contains a hydrated part, $\mathrm{mH}_{2} \mathrm{O}$ and another part with crystalline structure, $\mathrm{K}_{h} \mathrm{Fe}_{k}^{3+}\left\lfloor\mathrm{Fe}^{2+}(\mathrm{CN})_{6}\right\rfloor_{l}$. The $\mathrm{mH}_{2} \mathrm{O}$ part occupies interstitial sites which are located in channels of the hydrated structure $\mathrm{K}_{h} \mathrm{Fe}_{k}^{3+}\left[\mathrm{Fe}^{2+}(\mathrm{CN})_{6}\right]_{l}, m \mathrm{H}_{2} \mathrm{O}$. During the $\mathrm{PB} \leftrightarrow \mathrm{ES}$ transition, The $\mathrm{K}^{+}$movement is accompanied by the existing water molecules in the structure $\mathrm{K}_{h} \mathrm{Fe}_{k}^{3+}\left[\mathrm{Fe}^{2+}(\mathrm{CN})_{6}\right] . \mathrm{mH}_{2} \mathrm{O}$ although the $\mathrm{H}_{3} \mathrm{O}^{+}$movement occurs in the channels of this structure in order to maintain the film electroneutrality. This insertion mechanism of the cations $\mathrm{K}^{+}$and $\mathrm{H}_{3} \mathrm{O}^{+}$in independent sites is in agreement with the results obtained by ac-electrogravimetry and it is noticeable that no movement of free water through the film/solution interface was detected at low $\mathrm{pH}$ showing that water molecules stay in the film during the redox process. For higher $\mathrm{pH}$ water movement was demonstrated in agreement with results published in the literature $[49,50]$ In these conditions both water bounded to the protons and free water cross this interface. This difficulty of water transport at low $\mathrm{pH}$ is probably due to the fact that $\mathrm{PB}$ is an ionic crystalline solid, that is, a rigid structure [51] Obviously, this differs greatly from polymer electroactive film where the solvent molecules may contribute to the mass change during redox switching [52], particularly for polypyrrole [53] and polyaniline [38].

\section{6 - CONCLUSIONS}

The study of the PB film in a $\mathrm{KCl}$ medium at $\mathrm{pH} 2.5$ and 5.4 was carried out in this work. A theoretical model based on a porous film and which distinguishes electronic and ionic charge transfer was applied for the first time to interpret the experimental results obtained through ac-electrogravimetry.

The electrochemical impedance gave information on the global charge transfer kinetics which is controlled by ionic transfer for the whole potential range imposed to the $\mathrm{PB}$ film and whatever the $\mathrm{KCl}$ concentration as the resistance associated to the electronic transfer is lower than the resistance associated to the ionic transfer. However, electronic transfer becomes more and more limiting when the $\mathrm{KCl}$ concentration increases when the film behaviour is studied at $0.25 \mathrm{~V}$ vs. SCE. Moreover, the rates of the electronic and ionic transfer 
kinetics vary with the potential. They are faster around the potential where the $\mathrm{PB} \leftrightarrow \mathrm{ES}$ conversion occurs. The resistances associated to the electronic and ionic transfers present a minimum in this zone compared to the more anodic or negative potentials. The second information extracted from the impedance concerns the porosity of the PB film which is related to the zeolitic nature of the film. The resistivity of the pore depends on the potential and obviously on the electrolyte conductance.

$A c$-electrogravimetry gave essentially information on the kinetics of the ionic transfer. Thanks to the model of electroactive porous films and the digital fitting procedure, for the first time, the two cations $\mathrm{K}^{+}$and $\mathrm{H}_{3} \mathrm{O}^{+}$are identified with well separated kinetics for each cation. When a potential is applied to the $\mathrm{PB}$ film, the $\mathrm{H}_{3} \mathrm{O}^{+}$ion transfer is faster than the $\mathrm{K}^{+}$ ion transfer. Nevertheless, $\mathrm{K}^{+}$ions play a more important role than $\mathrm{H}_{3} \mathrm{O}^{+}$in the charge compensation process whatever the $\mathrm{pH}$ and the $\mathrm{KCl}$ concentration or the applied potential. However, the role of $\mathrm{H}_{3} \mathrm{O}^{+}$decreases for the higher $\mathrm{KCl}$ concentrations (between $0.1 \mathrm{M}$ and $0.5 \mathrm{M}$ ) when the film is studied at $\mathrm{pH}=5.4$ and for an imposed potential equal to $0.25 \mathrm{~V}$. Water movement was only detected for the highest $\mathrm{pH}$. For low $\mathrm{pH}$, water was blocked in the PB structure. Now, perspectives can be considered in term of structural changes which can accompanied the ion/solvent transfer. For that, a coupling between ac-electrogravimetry and XRD analysis seems an attractive direction to examine this idea. 


\begin{tabular}{|c|c|c|c|c|c|c|c|c|}
\hline $\begin{array}{c}\mathrm{C}_{\mathrm{M}} \\
\left(\mathrm{mole} \mathrm{l}^{-1}\right)\end{array}$ & $\log \left(c_{M}\right)$ & $\begin{array}{c}E_{a} \\
(\mathrm{~V})\end{array}$ & $\begin{array}{l}E_{c} \\
\text { (V) }\end{array}$ & $\begin{array}{l}E_{p-p} \\
\text { (V) }\end{array}$ & $\begin{array}{c}Q_{c} \\
\left(\mathrm{mC} \mathrm{cm}^{-2}\right)\end{array}$ & $\begin{array}{c}Q_{a} \\
\left(\mathrm{mC}_{2} \mathbf{c m}^{-}\right.\end{array}$ & $\begin{array}{c}\Delta m / \Delta E_{c} \\
\left(\mu \mathrm{cm}^{-2} \mathbf{V}^{-1}\right)\end{array}$ & $\begin{array}{c}\Delta m / \Delta E_{a} \\
\left(\mu \mathrm{g} \mathrm{cm} \mathbf{c m}^{-2} V^{-1}\right)\end{array}$ \\
\hline 0.01 & -2 & 0.177 & 0.011 & 0.094 & 4.177 & 4.092 & -0.584 & -3.061 \\
\hline 0.05 & -1.3 & 0.154 & 0.090 & 0.122 & 4.060 & 3.950 & -0.98 & -2.566 \\
\hline 0.1 & -1 & 0.16 & 0.116 & 0.138 & 3.963 & 3.861 & -1.266 & -2.804 \\
\hline 0.15 & \begin{tabular}{|c|}
-0.83 \\
\end{tabular} & 0.166 & 0.128 & 0.147 & 3.909 & 3.782 & $\begin{array}{l}-1.39 \\
\end{array}$ & -2.985 \\
\hline 0.25 & -0.6 & 0.168 & 0.134 & 0.151 & 3.856 & 3.690 & -1.535 & -3.099 \\
\hline 0.5 & -0.3 & 0.182 & 0.154 & 0.168 & 3.797 & 3.641 & -1.877 & -3.604 \\
\hline
\end{tabular}

Table 1: Parameters estimated from cyclic electrogravimetric curves with respect to the $\mathrm{KCl}$ concentration.

\begin{tabular}{|c|c|c|c|c|c|c|}
\hline $\begin{aligned} C_{M} \\
\mu \mathrm{cm}^{-2} V^{-1}\end{aligned}$ & 0.01 & 0.05 & 0.1 & 0.15 & 0.25 & 0.5 \\
\hline$\Delta \mathrm{m} / \Delta \mathrm{E}_{\mathrm{c}}$ & -0.584 & -0.98 & -1.266 & -1.39 & -1.535 & -1.877 \\
\hline$\left(\frac{\Delta m}{\Delta E}\right)_{0,025 H z}$ & -1.087 & -1.837 & -2.278 & -2.567 & -3.020 & -3.805 \\
\hline$\Delta \mathrm{m} / \Delta \mathrm{E}_{\mathrm{a}}$ & -3.061 & -2.566 & -2.804 & -2.985 & -3.099 & -3.604 \\
\hline
\end{tabular}

Table 2: $\left|\frac{\Delta m}{\Delta E}\right|$ values at $0.025 \mathrm{~Hz}$ of the PB film at $0.25 \mathrm{~V}$ vs. SCE. and comparison with $\Delta \mathrm{m} / \Delta \mathrm{E}$ obtained in cyclic electrogravimetry. 


\section{FIGURE CAPTIONS}

Figure 1: Diagram of the proposed porous model.

Figure 2: Cyclic voltammogram and electromassogram for a $\mathrm{PB}$ film in $0.5 \mathrm{M} \mathrm{KCl}$ at $\mathrm{pH} 2.5$ plotted for the $15^{\text {th }}$ cycle. Potential sweep between $-0.2 \mathrm{~V}$ and $0.6 \mathrm{~V}$ vs. SCE with a $20 \mathrm{mV} . \mathrm{s}^{-1}$ sweeping rate.

Figure 3: Experimental and theoretical transfer functions $(a, b, c)$ with the following estimated parameters at $0.175 \mathrm{~V}$ vs. SCE for a PB film in $0.5 \mathrm{M} \mathrm{KCl}$ at $\mathrm{pH} 2.5$.

Figure 4: Variations of $R_{e}, R_{t}(a), \rho_{2}(b), K_{c}(c), G_{c}(d), d C_{c}(E) / d E(e)$ and of the relative concentration of the species $\Delta \mathrm{C}_{\mathrm{c}}$ (f) with respect to the potential. Calculations were carried out from $\Delta E / \Delta I(\omega)$ and $\Delta m / \Delta E(\omega)$ functions for a $\mathrm{PB}$ film in $0.5 \mathrm{M} \mathrm{KCl}$ at $\mathrm{pH} 2.5$.

Figure 5: Cyclic electrogravimetry of the redox behaviour of a PB film (a) in $0.05 \mathrm{M} \mathrm{KCl}$ at pH 5.4 obtained at the $10^{\text {th }}$ cycle with a potential scan between $-0.2 \mathrm{~V}$ and $0.6 \mathrm{~V}$ vs. SCE and a $20 \mathrm{mV} \cdot \mathrm{s}^{-1}$ sweep rate.

Figure 6: Variation with respect to the potential of $\mathrm{K}_{\mathrm{c}}(\mathrm{a}) \mathrm{G}_{\mathrm{c}}(\mathrm{b}), \mathrm{dC}(\mathrm{E}) / \mathrm{dE}$ (c) and of the change of the concentrations of species $\Delta \mathrm{C}_{\mathrm{c}}$ in the PB film (d) calculated from the transfer functions $\Delta E / \Delta I(\omega)$ and $\Delta m / \Delta E(\omega)$ for a PB film in $0.05 \mathrm{M} \mathrm{KCl}$ at $\mathrm{pH} 5.4$.

Figure 7: Cyclic electrogravimetric curves: $i-E$ (a) and $\Delta m-E$ (b), for a PB film cycled between -0.2 and $0.6 \mathrm{~V}$ vs. SCE with a $20 \mathrm{mV} \mathrm{s}^{-1}$ sweeping rate, for various $\mathrm{KCl}$ concentrations.

Figure 8: Diagrams of the electrochemical impedance (a) and variations of $R_{e}$ and $R_{t}$ (b), $R_{t} / R_{e}$ (c), $\rho_{2}$ and $R_{s}(\mathrm{~d})$ with respect to the $\mathrm{KCl}$ concentration for a $\mathrm{PB}$ film immersed in an $\mathrm{pH} 5.4$ aqueous medium at $0.25 \mathrm{~V}$ vs SCE. 
Figure 9: Electrogravimetric transfer function (a) and variation of $\mathrm{Kc}(\mathrm{b}), \mathrm{Gc}(\mathrm{c})$ and $d C_{c} / d E$ at $0.25 \mathrm{~V}$ vs. $\mathrm{SCE}$, with respect to the $\mathrm{KCl}$ concentration at $\mathrm{pH} 5.4$. 


\section{REFERENCES}

${ }^{1}$ D. Ellis, M. Eckhoff; V. D. Neff, J. Phys. Chem. 85 (1981) 1225.

${ }^{2}$ K. Itaya, H. Akahoshi, S. Toshima, J. Electrochem. Soc., 129 (1982) 762.

${ }^{3}$ K. Itaya, T. Ataka; S. J. Toshima, J. Am.Chem.Soc., 104 (1982) 3751.

${ }^{4}$ H. Kellawi, D. R. Rosseinsky, J. Electroanal. Chem., 131 (1982) 373.

${ }^{5}$ V. D. Neff, 125 (1978) 886.

${ }^{6}$ J. F. Keggin; F. D. Miles, 137 (1936) 577.

${ }^{7}$ H. J. Buser, D. Schwarzenbach, W. Petter, A. Ludi, Inorg. Chem. 16 (1977) 2704.

${ }^{8}$ F. Herren, P. Fischer, A. Ludi, W. Haelg, Inorg.Chem., 19 (1980) 956.

${ }^{9}$ B. Bal; S. Ganguli, M. Bhattacharya, J. Phys.1 Chem., 88 (2002) 4575.

${ }^{10}$ K. Itaya, T. Ataka, S. Toshima,. J.Am.Chem.Soc. 104 (1982) 4767.

11 J. J. Garcia-Jareno, A. Sanmatias, F. Vicente, C. Gabrielli, M. Keddam, H. Perrot, Electrochim.Acta, 45 (2000) 3765.

${ }^{12}$ B. J. Feldman, O. R. Melroy, J. Electroanal. Chem. 234 (1987) 213.

${ }^{13}$ V. Plichon, S. Besbes, J. Electroanal. Chem., 284 (1990) 141.

${ }^{14}$ J. J. Garcia-Jarero, A. Sanmatias, J. Navarro-Laboulais, F. Vicente, Electrochim. Acta, 44 (1998) 395.

15 J. J. Garcia-Jareno, J. Navarro-Laboulais, F. Vicente, Electrochim. Acta, 41 (1996) 835.

${ }^{16}$ K. Kim, I. Jureviciute, S. Bruckenstein, Electrochim. Acta, 46 (2001) 4133.

${ }^{17}$ C. Gabrielli, J. J. Garcia-Jareno, M. Keddam, H. Perrot, F. Vicente, J. Phys. Chem. B, 106 (2002) 3182.

${ }^{18}$ C. Debiemme-Chouvy, A. Rubin, H. Perrot, C. Deslouis and H. Cachet, Electrochimica Acta., 53, 11 (2008) 3836.

${ }^{19}$ L. To Thi Kim, O. Sel, C. Debiemme-Chouvy, C. Gabrielli, C. Laberty-Robert, H. Perrot and C. Sanchez, Electrochem. Com., 12(2010)1136-1139.

${ }^{20}$ J. Euler, W. Nonnenmacher, Electrochim. Acta, 2 (1960) 268.

${ }^{21}$ R. de Levie, Electrochim. Acta, 8 (1963) 751.

${ }^{22}$ R. de Levie, Electrochim. Acta, 9 (1964) 1231.

${ }^{23}$ R. de Levie, Electrochim. Acta, 10 (1965) 113.

${ }^{24}$ M. Keddam, C. Rakotomavo, H. Takenouti, J. Applied Electrochem., 14 (1984) 437.

${ }^{25}$ J. S. Newman, C. W. Tobias, J. Electrochem. Soc., 109 (1962) 1183.

${ }^{26}$ C. Cachet, R. Wiart, J. Electroanal. Chem., 195 (1985) 21.

${ }^{27}$ O. Bohnke, B. Vuillemin, C. Gabrielli, M. Keddam, H. Perrot, Electrochim.Acta 40 (1995) 2765-2773.

${ }^{28}$ F. La Mantia, J. Vetter, P. Novak, Electrochim. Acta, 53 (2008) 4109.

${ }^{29}$ G. C. Barker, J. Electroanal. Chem. 41 (1973) 201.

${ }^{30}$ W. J. Albery, C. M. Elliott, A. R. Mount, J. Electroanal. Chem., 288 (1990) 15.

${ }^{31}$ R. P. Buck, C. Mundt, J.Chem.Soc., Faraday Trans., 92 (1996) 3947.

${ }^{32}$ T. R. Brumleve, R. P. Buck, J. Electroanal. Chem., 126 (1981) 73.

${ }^{33}$ K. Rosberg, G. Paasch, L. Dunsch; S. Ludwig, J. Electroanal. Chem., 443 (1998) 49. 
${ }^{34}$ P. H. Nguyen, G. Paasch, J. Electroanal. Chem., 460 (1999) 63.

${ }^{35}$ G. Paasch, J. Electroanal. Chem., 600 (2007) 131.

${ }^{36}$ C. Ehrenbeck, K. Juttner, S. Ludwig, G. Paasch, Electrochim. Acta, 43 (1998) 2781-2789.

${ }^{37}$ C. Gabrielli, J. J. Garcia-Jareno,H. Perrot, Electrochim. Acta, 16 (2001) 4095.

${ }^{38}$ C. Gabrielli, M. Keddam, N. Nadi, H. Perrot, J. Electroanal. Chem., 485 (2000) 101.

39 A. Jackson, A. R. Hillman, S. Bruckenstein, I. Jureviciute, J. Electroanal. Chem., 524 (2002) 90.

${ }^{40}$ J. P. Candy, P. Fouilloux, M. Keddam, H. Takenouti, Electrochim. Acta, 27 (1982) 1585.

${ }^{41}$ T. T. L. Kim, PhD thesis, Univ. P. and M. Curie, Paris, France, (2009).

${ }^{42}$ C. Gabrielli, H. Perrot, in Modern aspects of electrochemistry, Vol. 44, p.151-238.

${ }^{43}$ J.J. Garcia-Jareno, C. Gabrielli, and H. Perrot, Electrochem. Com., 2(2000)195-200.

${ }^{44}$ D. Gimenez-Romero, P. R. Bueno, C. Gabrielli, J. J. Garcia-Jareno, H. Perrot, F. Vicente, J. Phys. Chem. B, 110 (2006) 19352.

${ }^{45}$ C. L. Lin, K. C. Ho, J. Electroanal. Chem., 524-525 (2002) 286.

${ }^{46}$ A. Dostal, G. Kauschka, S. J. Reddy, F. Scholz, J. Electroanal. Chem., 406 (1996) 155.

${ }^{47}$ I. Oh, H. Lee, H. Yang, J. Kwak, Electrochem. Comm., 3 (2001) 274.

${ }^{48}$ D. Gimenez-Romero, P. R. Bueno, J. J. Garcia-Jareno, C. Gabrielli, H. Perrot, F. Vicente,.J. Phys. Chem. B, 110 (2006) 2715.

${ }^{49}$ S. J. Lasky, D. A. Buttry, J. Am. Chem. Soc., 110 (1988) 6258.

${ }^{50}$ K. Ogura, M. Nakayama, K. Nakaoka, J. Electroanal. Chem., 474 (1999) 101.

${ }^{51}$ D. Gimenez-Romero, P. R. Bueno, J. J. Garcia-Jareno, C. Gabrielli, H. Perrot, F. Vicente, J. Phys. Chem. B, 110 (2006) 2715.

${ }^{52}$ M. Henderson, A. R. Hillman, E. Vieil, J. Phys.Chem. B, 103 (1999) 8899.

${ }^{53}$ C. Gabrielli, J. J. Garcia-Jareno, M. Keddam, H. Perrot, F. Vicente, J. Phys. Chem. B, 106 (2002) 3192. 
- New model of ionic/solvent insertion in electroactive films is developed including porosity.

- Coupling electrochemical impedance and fast quartz crystal microbalance appears as an attractive and powerful technique to investigate electroactive films.

- The separation between potassium and hydronium ions is now possible on PB films. 


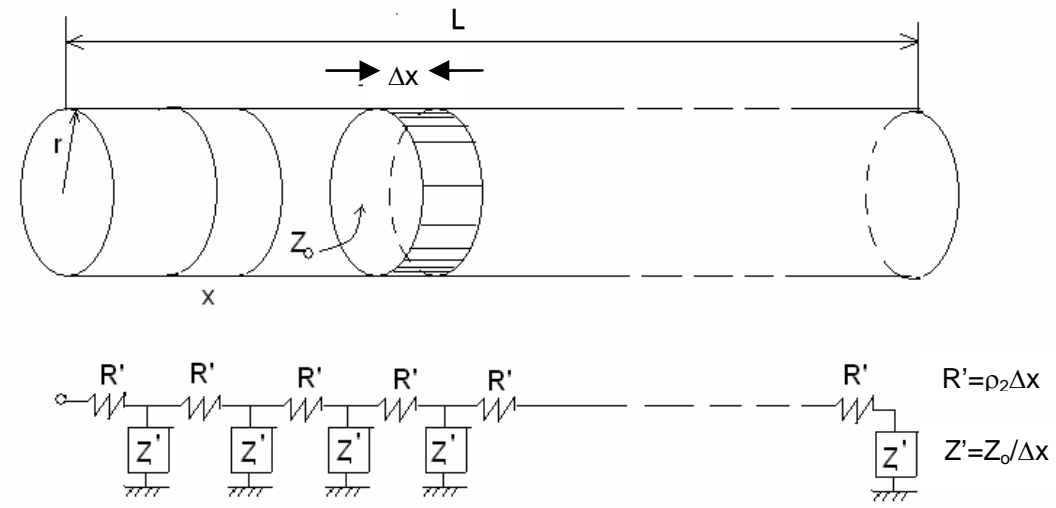




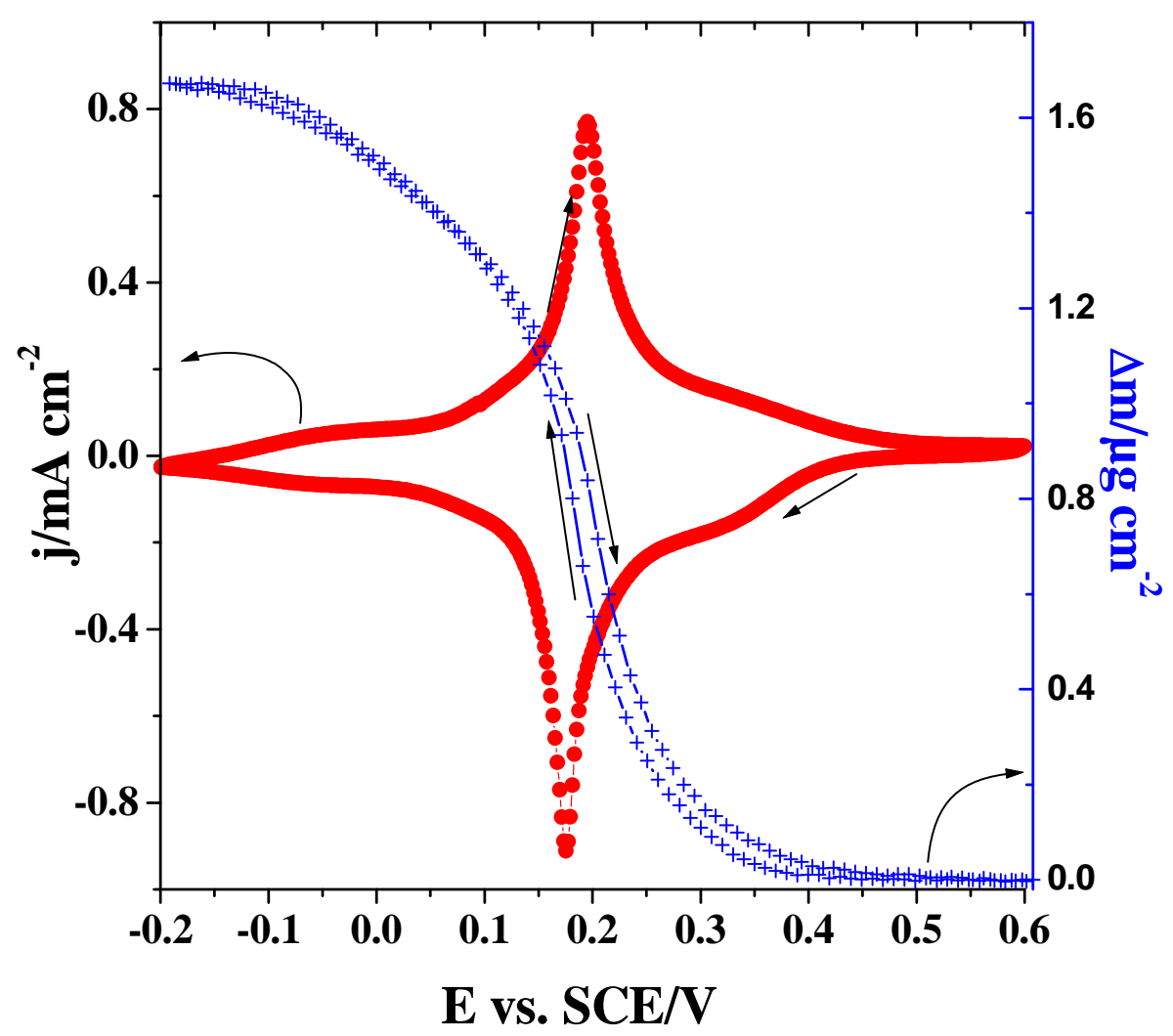




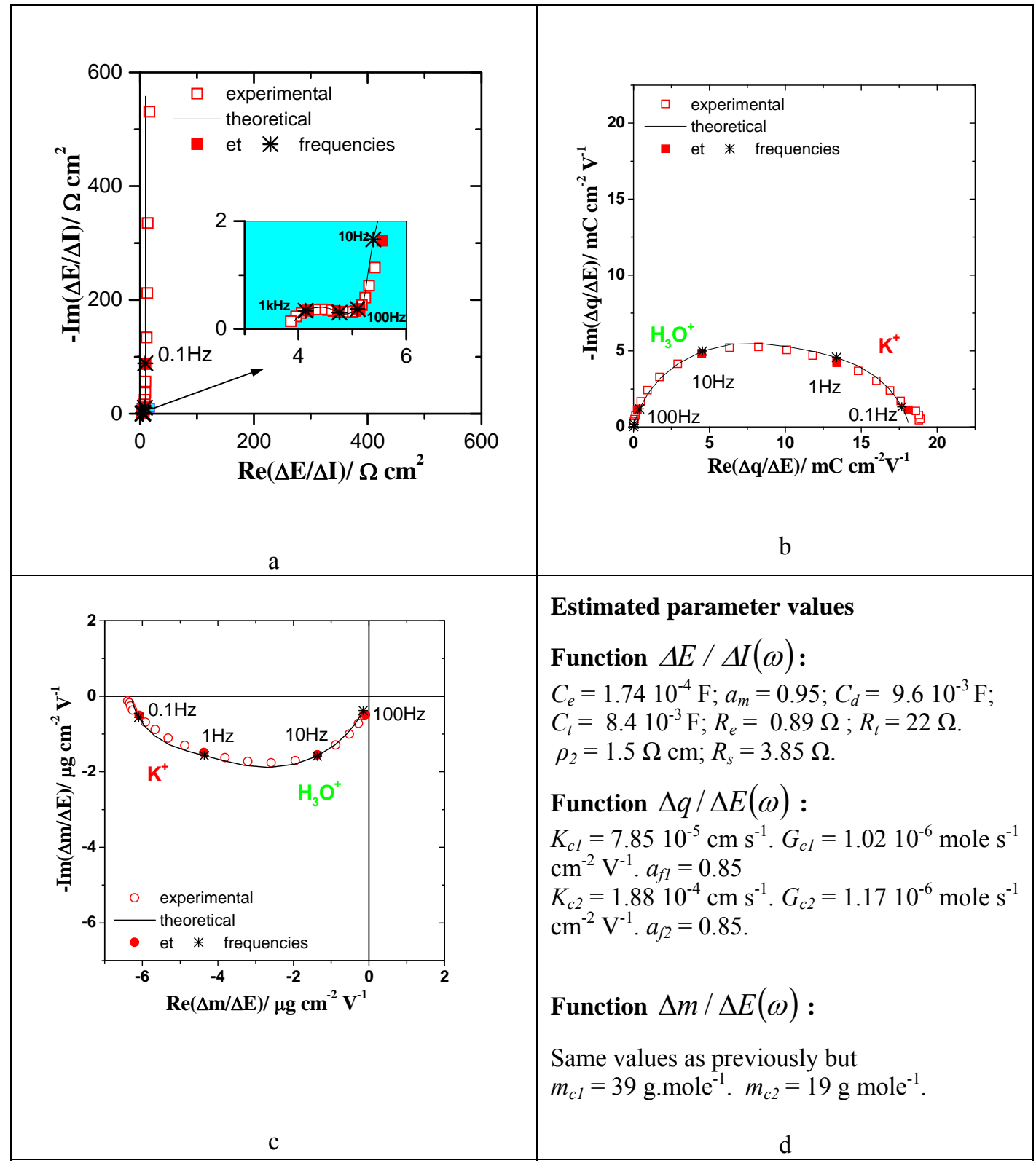




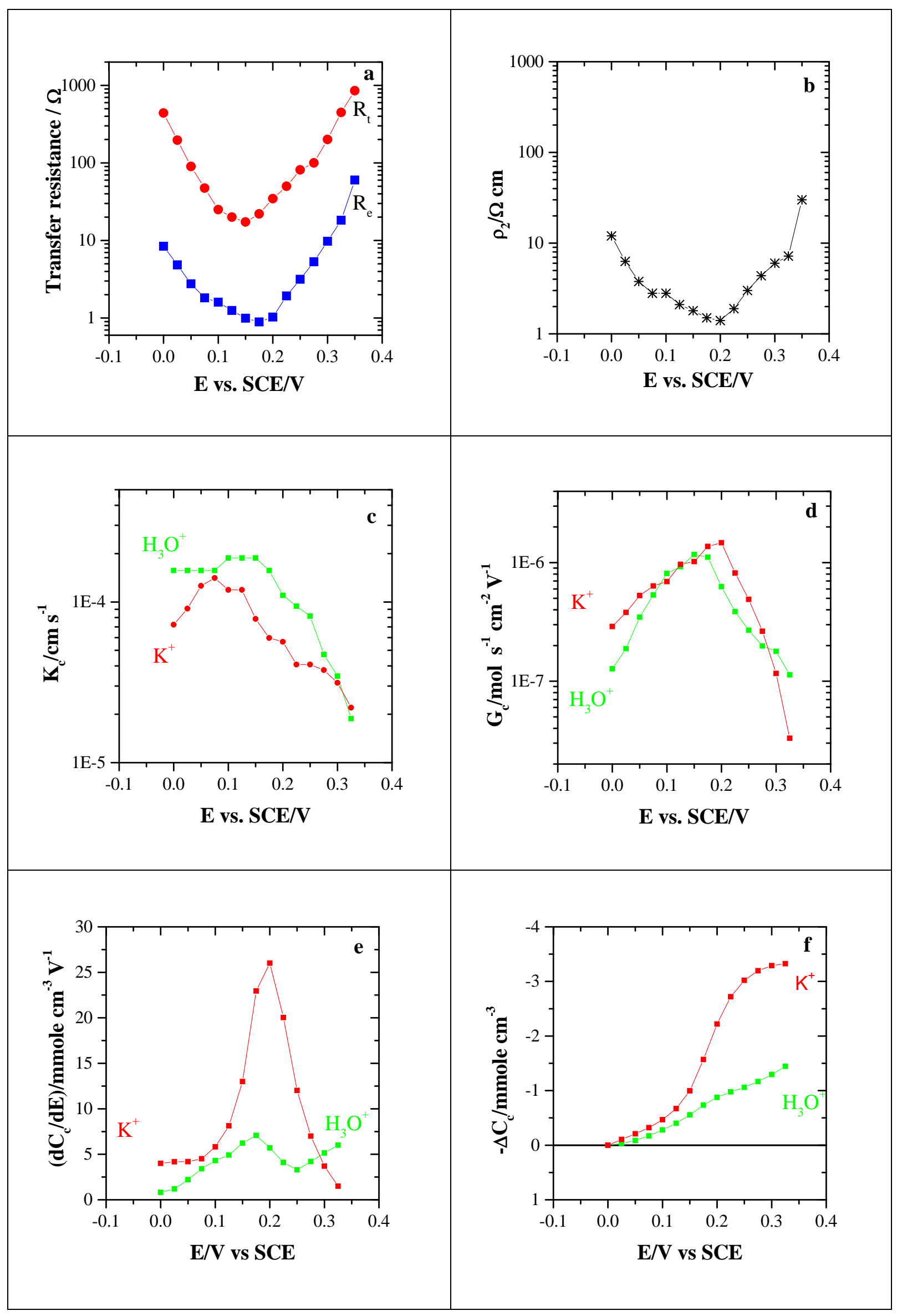




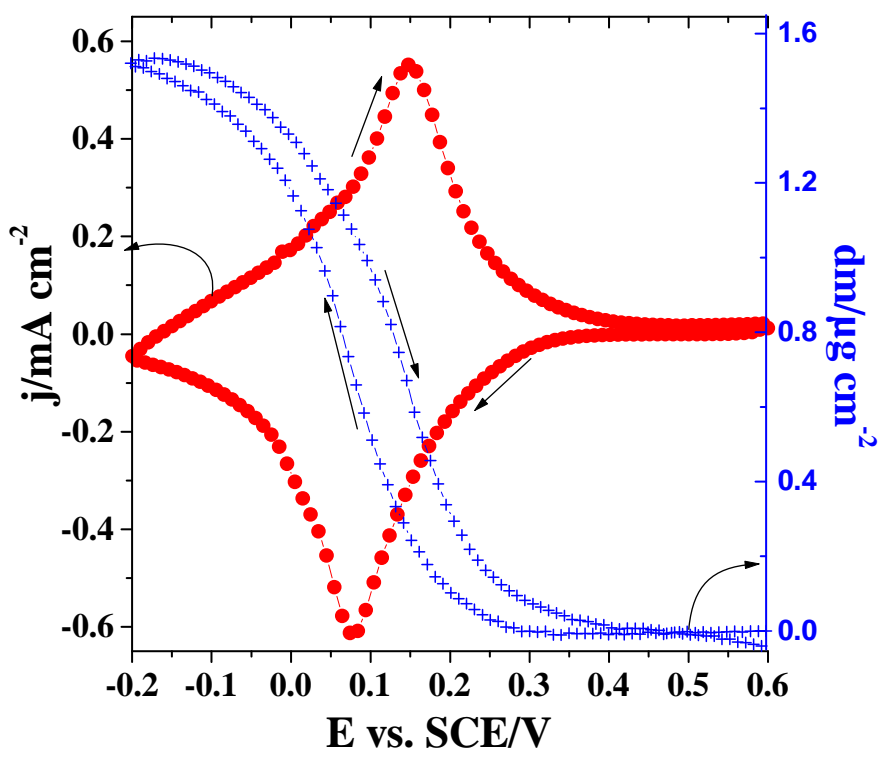




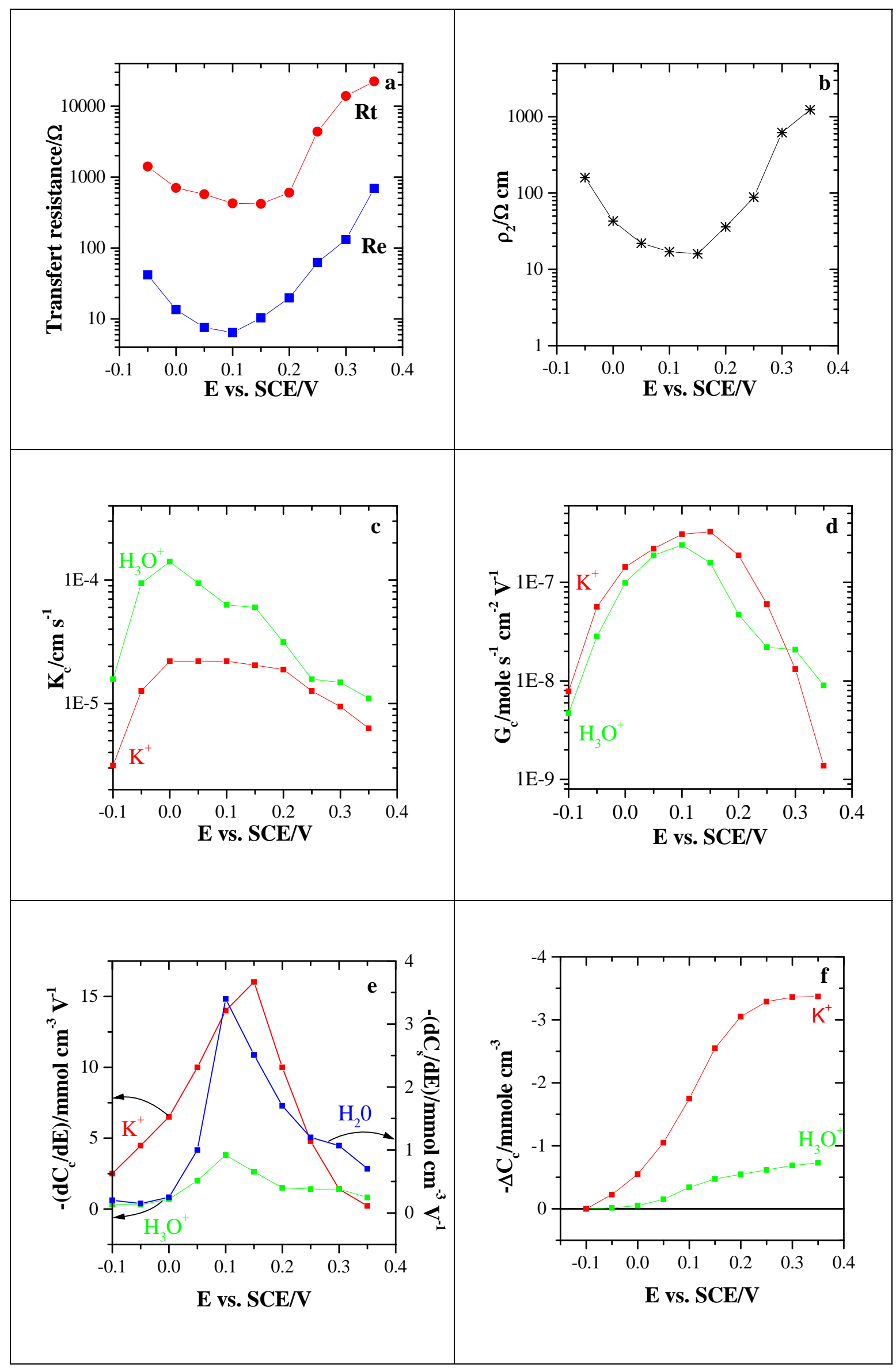




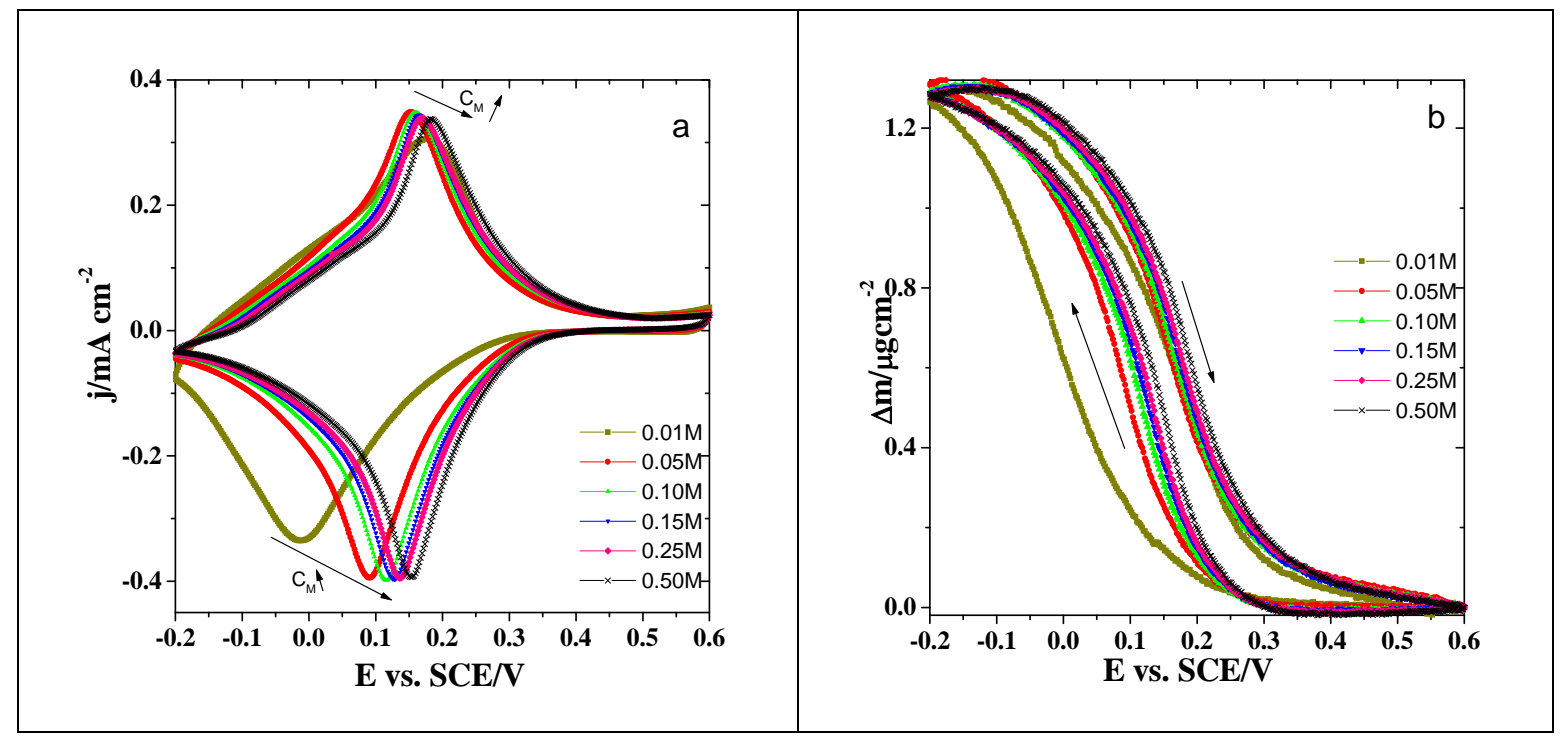




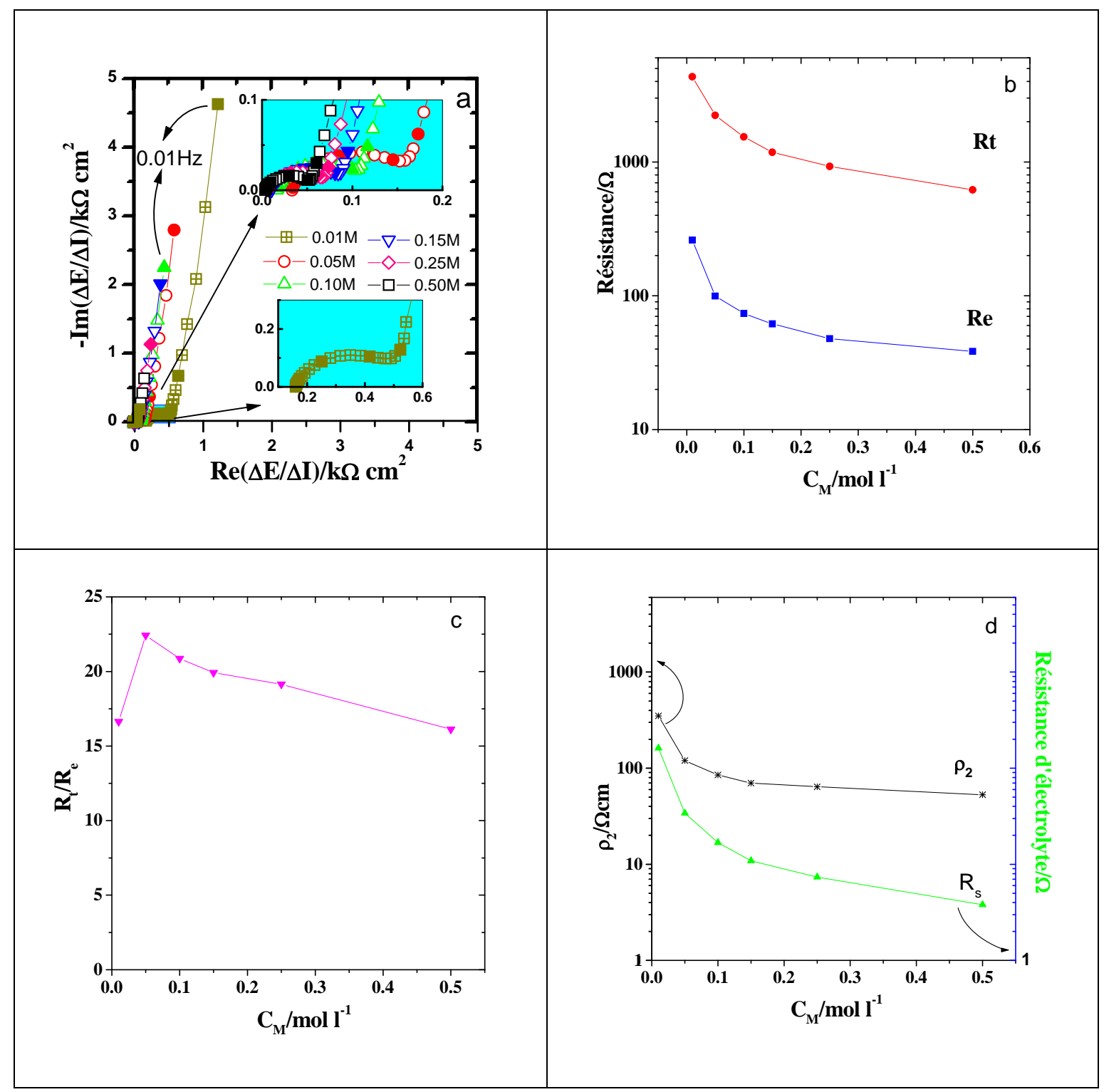




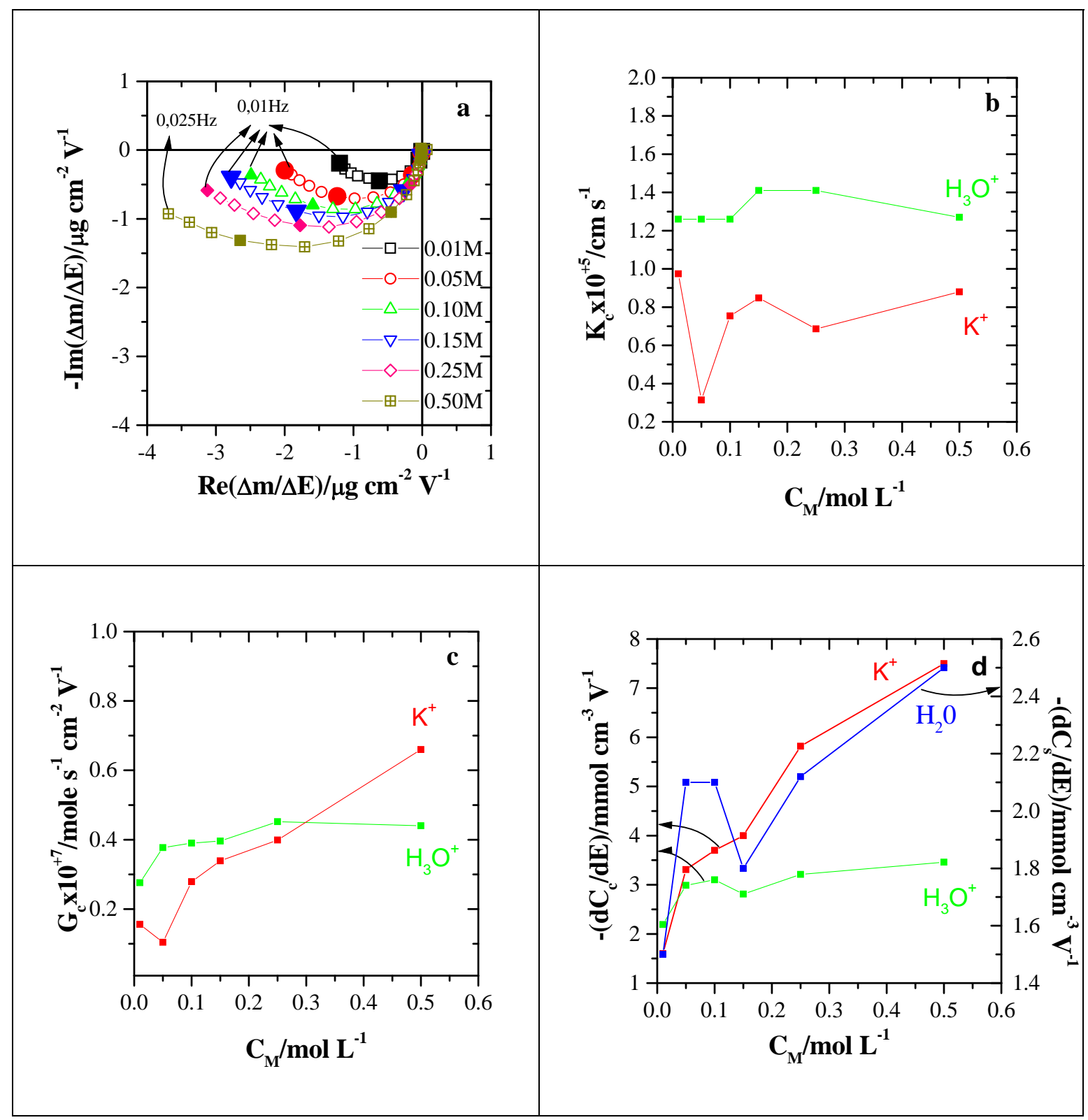

